JOURNAL OF THE

AMERICAN MATHEMATICAL SOCIETY

Volume 23, Number 1, January 2010, Pages 267-297

S 0894-0347(09)00646-8

Article electronically published on October 1, 2009

\title{
STABLE PAIRS AND BPS INVARIANTS
}

\author{
R. PANDHARIPANDE AND R. P. THOMAS
}

\section{Contents}

0. Introduction . . . . . . . . . . . . . . . . . . 268

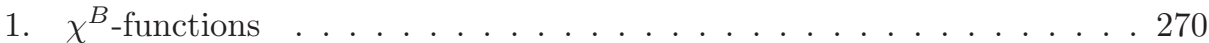

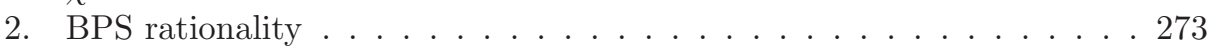

2.1. Results . . . . . . . . . . . . . . . . . . 273

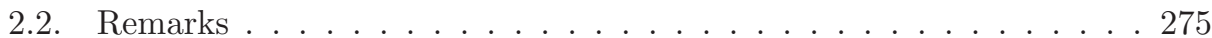

2.3. Wall-crossing . . . . . . . . . . . . . . . 275

3. Local definition of BPS invariants . . . . . . . . . . . . . . . 276

3.1. Fixed curve . . . . . . . . . . . . . . . . . . 276

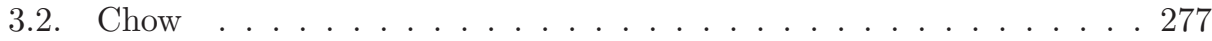

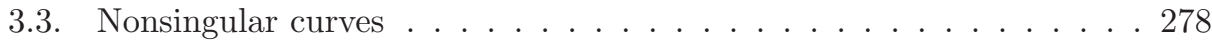

3.4. Singular curves: Discussion . . . . . . . . . . . . . . . . 279

3.5. Singular curves: Results . . . . . . . . . . . . . . . . . . . 280

3.6. Nodal curves . . . . . . . . . . . . . . . . . . . . . 284

Appendix A. Reduced curve classes . . . . . . . . . . . . . . . . . 287

Appendix B. Stable pairs on Gorenstein curves . . . . . . . . . . . . . . . 289

B.1. Gorenstein curves . . . . . . . . . . . . . . . . . . . . . . 289

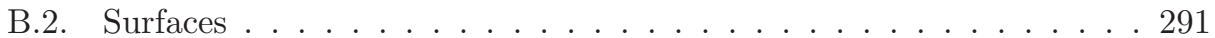

Appendix C. K3 surfaces ................... 292

C.1. Nonsingularity . . . . . . . . . . . . . . . . . . . . 292

C.2. BPS states ... . . . . . . . . . . . . . . . 294

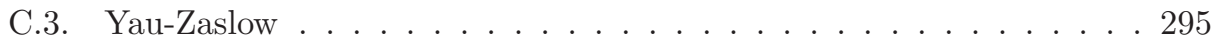

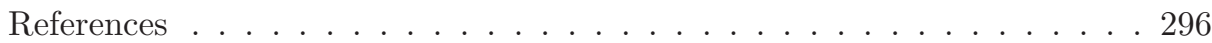

Received by the editors October 14, 2008.

2000 Mathematics Subject Classification. Primary 14N35.

The first author was partially supported by NSF grant DMS-0500187 and a Packard foundation fellowship.

The second author was partially supported by a Royal Society University Research Fellowship. He also thanks the Leverhulme Trust and Columbia University for a visit to New York in the spring of 2007 when the project was started. Many of the results presented here were found during a visit to Lisbon in the summer of 2007.

(C)2009 American Mathematical Society Reverts to public domain 28 years from publication 


\section{INTRODUCTION}

Let $X$ be a nonsingular, projective, Calabi-Yau 3-fold 1 Invariants counting curves in $X$ via stable pairs have been defined in [26]. A pair $(F, s)$ consists of a sheaf $F$ on $X$ supported in dimension 1 together with a section $s \in H^{0}(X, F)$. A pair $(F, s)$ is stable if

(i) the sheaf $F$ is pure,

(ii) the section $\mathcal{O}_{X} \stackrel{s}{\rightarrow} F$ has 0-dimensional cokernel.

By purity (i), every nonzero subsheaf of $F$ has support of dimension 1 . As a consequence, the scheme-theoretic support $C \subset X$ of $F$ is a Cohen-Macaulay curve. The support of the cokernel (ii) is a finite length subscheme $Z \subset C$. If the support $C$ is nonsingular, then the stable pair $(F, s)$ is uniquely determined by $Z \subset C$. However, for general $C$, the subscheme $Z$ does not determine $F$ and $s$.

Discrete invariants of a stable pair include the holomorphic Euler characteristic $\chi(F) \in \mathbb{Z}$ and the class $[F] \in H_{2}(X, \mathbb{Z})$. The moduli space $P_{n}(X, \beta)$ parameterizes stable pairs satisfying

$$
\chi(F)=n, \quad[F]=\beta .
$$

Pair stability arises naturally as GIT stability in the study of appropriate quotients 20, 26. In fact, GIT stability is found there to be equivalent to semi-stability. The moduli space $P_{n}(X, \beta)$ is therefore a projective scheme.

To define invariants, we use a virtual cycle. The usual deformation theory of pairs is not perfect in the sense of 4, but the fixed-determinant deformation theory of the associated complex

$$
I^{\bullet}=\left\{\mathcal{O}_{X} \stackrel{s}{\longrightarrow} F\right\} \in D^{b}(X)
$$

is shown in [26, 13] to define a perfect obstruction theory for $P_{n}(X, \beta)$ of virtual dimension zero. A virtual cycle is then obtained by [4, 22]. The resulting invariants

$$
P_{n, \beta}=\int_{\left[P_{n}(X, \beta)\right]^{v i r}} 1
$$

are conjecturally equal to the reduced DT invariants of 23$]$. Let

$$
Z_{\beta}(q)=\sum_{n \in \mathbb{Z}} P_{n, \beta} q^{n}
$$

be the generating series. Calculations in the toric Calabi-Yau case can be found in 27.

Since $X$ is Calabi-Yau, the above deformation theory of complexes is self-dual in the sense of [3]. Heuristically, $P_{n}(X, \beta)$ may be viewed locally as the critical locus of a function. The virtual dimension of $P_{n}(X, \beta)$ is 0 and, on the nonsingular locus of the moduli space, the obstruction sheaf is the cotangent bundle. Therefore if $P_{n}(X, \beta)$ is everywhere nonsingular, then

$$
P_{n, \beta}=(-1)^{\operatorname{dim} P_{n}(X, \beta)} e\left(P_{n}(X, \beta)\right),
$$

where $e$ denotes the topological Euler characteristic.

If singularities are present, $P_{n, \beta}$ certainly differs from the (signed) Euler characteristic. By Behrend's results [3], there exists an integer-valued constructible function $\chi^{B}$ over any scheme with the property that if the scheme is proper and

\footnotetext{
${ }^{1}$ The Calabi-Yau condition for us is $K_{X} \cong \mathcal{O}_{X}$. No restriction of the fundamental group is necessary.
} 
admits a self-dual obstruction theory, then the length of its virtual cycle equals its $\chi^{B}$-weighted Euler characteristic. Therefore

$$
P_{n, \beta}=e\left(P_{n}(X, \beta), \chi^{B}\right):=\sum_{n \in \mathbb{Z}} n e\left(\left(\chi^{B}\right)^{-1}(n)\right) .
$$

At nonsingular points,

$$
\chi^{B}=(-1)^{\operatorname{dim} P_{n}(X, \beta)},
$$

but at singularities $\chi^{B}$ is a more complicated function. The weighted Euler characteristic of $P_{n}(X, \beta)$ is a deformation invariant.

Behrend's theory applied to $P_{n}(X, \beta)$ allows us to use topological Euler characteristics and cut-and-paste techniques. We require new technical results comparing the value of Behrend's function at a pair $(F, s)$ to the value at the sheaf $F$; see Theorem 4 of Section 1 . The arguments turn out to be remarkably simple when $\beta$ is an irreducible 2 class. We prove the following result in Section 2

Theorem 1. For $\beta$ irreducible, $Z_{\beta}(q)$ is the Laurent series expansion of a rational function in $q$.

Serre duality relates a line bundle $L$ on a nonsingular curve $C \subset X$ to $L^{-1} \otimes K_{C}$. Since

$$
\chi(L)=-\chi\left(L^{-1} \otimes K_{C}\right),
$$

Serre duality relates the geometry of $P_{n}(X, \beta)$ to $P_{-n}(X, \beta)$. The compatibility of Serre duality with $\chi^{B}$ proven in Section 2 yields a more subtle result.

Theorem 2. For $\beta$ irreducible, the rational function $Z_{\beta}(q)$ is invariant under the transformation $q \leftrightarrow q^{-1}$.

In fact, we prove that $Z_{\beta}(q)$ satisfies the full BPS rationality conjectured in [26].

Theorem 3. For $\beta$ irreducible,

$$
Z_{\beta}(q)=\sum_{r=0}^{g} n_{r, \beta} q^{1-r}(1+q)^{2 r-2},
$$

where the $n_{r, \beta}$ are integers and $g$ is the maximal arithmetic genus $g$ of a curve in class $\beta$.

We obtain a deformation-invariant definition of the BPS counts $n_{r, \beta}$ of Gopakumar-Vafa 7, 8, for irreducible classes $\beta$. In Section 3, we give a local definition of these BPS invariants for irreducible curve classes. We define constructible functions over the space of curves in $X$ with respect to which the weighted Euler characteristics yield the BPS numbers. We prove that the functions are nonzero on $C \subset X$ only in genus $g$ between the geometric and arithmetic genera of $C$. Complete evaluations of the functions are obtained for nonsingular and nodal curves.

In Appendix A, we sketch the extension of Theorems 1 and 2 to reduced curve classes which are not necessarily irreducible. We also explain what is needed to show the vanishing of BPS counts in negative genus in the reduced case.

Interesting examples of irreducible and reduced classes occur on surfaces. If $C$ is Gorenstein, the stable pairs with support $C$ are proven in Appendix B to correspond bijectively to finite length subschemes $Z \subset C$. The moduli spaces of stable pairs on a surface are then shown to be isomorphic to relative Hilbert schemes.

\footnotetext{
${ }^{2}$ The definitions of irreducible, primitive, and reduced curve classes are given in Section 1.
} 
In Appendix C, the beautiful theory of primitive classes on $K 3$ surfaces is considered. By results of Kawai-Yoshioka [17, the Katz-Klemm-Vafa [16] formula for BPS state counts is obtained for the theory of stable pairs. The corresponding calculations in Gromov-Witten theory have not yet been completed 3

Let $r_{0, g}$ be the number of rational curves of fixed primitive class with selfintersection $2 g-2$ on a $K 3$ surface. Using the genus 0 BPS counts together with the local BPS theory of Section 3, a new proof of the Yau-Zaslow formula,

$$
\sum_{g \geq 0} r_{0, g} q^{g}=\prod_{n \geq 0}\left(1-q^{n}\right)^{-24},
$$

is obtained.

The Yau-Zaslow formula was proven in the primitive 4 case by Bryan-Leung [5] via Gromov-Witten theory. Our proof is very close in spirit to the original sheaftheoretic motivations for the formula [34. In particular, our argument via stable pairs and BPS counts is parallel to Beauville's proof using compactified Jacobians and Euler characteristics 2 .

\section{1. $\chi^{B}$-FUnCTIONS}

Let $X$ be a nonsingular projective variety over $\mathbb{C}$. A nonzero class $\beta \in H_{2}(X, \mathbb{Z})$ is effective if $\beta$ is represented by an algebraic curve.

Definition 1.1. An effective class $\beta \in H_{2}(X, \mathbb{Z})$ is

- irreducible if there is no decomposition $\beta=\beta_{1}+\beta_{2}$ into nonzero effective classes $\beta_{i}$,

- primitive if $\beta$ is not a positive integer multiple of an effective class,

- reduced if in every decomposition $\beta=\sum_{i} \beta_{i}$ into effective classes, all of the $\beta_{i}$ are primitive.

For example, classes $\beta$ of minimal degree $\int_{\beta} c_{1}(L)$ measured against any ample class $L$ are irreducible. Any primitive class $\beta$ on a $K 3$ surface $S$ is irreducible on a generic deformation of $S$ for which $\beta$ is of type $(1,1)$.

Let $X$ be a Calabi-Yau 3-fold. If $(F, s)$ is a a stable pair of irreducible class $\beta$, then $F$ is a stable sheaf since all quotient sheaves have 0-dimensional support 5 There is therefore a map

$$
P_{n}(X, \beta) \stackrel{\phi_{n}}{\longrightarrow} \mathcal{M}_{n}(X, \beta)
$$

from the moduli space of stable pairs to the moduli space of stable pure sheaves of the Hilbert polynomial

$$
\chi(F(k))=k \int_{\beta} c_{1}(L)+n .
$$

Moreover, the fibre of (1.2) over a point $\{F\}$ is $\mathbb{P}\left(H^{0}(F)\right)$. By the irreducibility of $\beta$, the cokernel of any nonzero $s$ section is 0 -dimensional and $(F, s)$ is a stable pair.

Since $X$ is Calabi-Yau, both $P_{n}(X, \beta)$ [26] and $\mathcal{M}_{n}(X, \beta)$ [31 have self-dual obstruction theories. We can therefore apply the results of [3].

\footnotetext{
${ }^{3} \mathrm{~A}$ discussion of the Gromov-Witten side can be found in [24].

${ }^{4} \mathrm{~A}$ proof of the Yau-Zaslow formula for all curve classes on $K 3$ surfaces has been recently obtained in [18.

${ }^{5}$ Usually, sheaf stability depends upon the polarization $L$. However, for sheaves $F$ of rank 1 on an irreducible curve in $X$, stability is equivalent to purity, and, no strictly semi-stable sheaves exist.
} 
Lemma 1.3. The obstruction theory of $P_{n}(X, \beta)$ obtained from fixed determinant deformations in the derived category [26] is self-dual in the sense of Behrend [3].

Proof. The obstruction theory of [26] can be described as follows. Let

$$
\pi: X \times P_{n}(X, \beta) \rightarrow P_{n}(X, \beta)
$$

be the projection. There is a universal stable pair [26,

$$
\mathcal{O}_{X \times P_{n}(X, \beta)} \rightarrow \mathbb{F},
$$

over $X \times P_{n}(X, \beta)$. Let $\mathbb{I} \bullet$ be the associated complex (with $\mathcal{O}_{X \times P_{n}(X, \beta)}$ in degree $0)$. Consider the complex

$$
R \pi_{*} R \mathscr{H} O \mathrm{om}\left(\mathbb{I}^{\bullet}, \mathbb{I}^{\bullet} \otimes \omega_{\pi}\right)_{0}[2]
$$

of trace-free Exts, where $\omega_{\pi}$ denotes the relative canonical bundle. In [26, the complex (1.4) is shown to be quasi-isomorphic to a 2-term complex of locally free sheaves $\left\{E_{1} \rightarrow E_{0}\right\}$ over $P_{n}(X, \beta)$, with a canonical morphism

$$
R \pi_{*} R \mathscr{H} O \mathrm{om}\left(\mathbb{I}^{\bullet}, \mathbb{I} \bullet \otimes \omega_{\pi}\right)_{0}[2] \rightarrow L_{P_{n}(X, \beta)}^{\bullet}
$$

to the cotangent complex of $P_{n}(X, \beta)$. The morphism is obstruction theory for $P_{n}(X, \beta)$ : the induced maps on $h^{0}$ and $h^{1}$ are isomorphisms and surjections respectively.

For $X$ Calabi-Yau, $\omega_{\pi}$ is trivial. Therefore, by relative Serre duality for $\pi$, we obtain a quasi-isomorphism

$$
R \pi_{*} R \mathscr{H} \mathrm{om}\left(\mathbb{I}^{\bullet}, \mathbb{I}^{\bullet}\right)_{0}^{\vee} \simeq R \pi_{*} R \mathscr{H} \mathrm{om}\left(\mathbb{I}^{\bullet}, \mathbb{I}^{\bullet}\right)_{0}[3] .
$$

Thus

$$
\left\{E_{0}^{\vee} \rightarrow E_{1}^{\vee}\right\}[1] \simeq\left\{E_{1} \rightarrow E_{0}\right\},
$$

which is the definition of self-duality in [3].

For any scheme $M$, Kai Behrend $[3]$ defines a canonical constructible function

$$
\chi^{B}: M \rightarrow \mathbb{Z},
$$

depending only on the local scheme structure 6 If $M$ is compact and equipped with a self-dual obstruction theory, then

$$
\int_{[M]^{v i r}} 1=e\left(M, \chi^{B}\right),
$$

where the right side is the weighted Euler characteristic

$$
e\left(M, \chi^{B}\right)=\sum_{n \in \mathbb{Z}} n e\left(\left(\chi^{B}\right)^{-1}(n)\right)
$$

and $e$ is the usual topological Euler characteristic.

If $M$ is nonsingular, then $\chi^{B}$ is the constant function $(-1)^{\operatorname{dim} M}$ and

$$
\int_{[M]^{v i r}} 1=(-1)^{\operatorname{dim} M} e(M)
$$

More generally, by Proposition 1.5(i) of [3], if $f: M \rightarrow N$ is a smooth map of relative dimension $r$, then

$$
\chi_{M}^{B}=(-1)^{r} f^{*} \chi_{N}^{B} .
$$

\footnotetext{
${ }^{6}$ In fact, $\chi^{B}$ depends only on the local scheme structure in analytic topology by Proposition 4.22 of 3 .
} 
If $e(F(f))$ is the Euler characteristic of the fibre of $f$, then

$$
e\left(M, \chi_{M}^{B}\right)=(-1)^{r} e\left(N, \chi_{N}^{B}\right) \cdot e(F(f)) .
$$

On $P_{n}(X, \beta)$ and $\mathcal{M}_{n}(X, \beta)$, we obtain functions $\chi_{P}$ and $\chi_{\mathcal{M}} 7$ The invariants

$$
\begin{aligned}
P_{n, \beta} & =\int_{\left[P_{n}(X, \beta)\right]^{\text {vir }}} 1=e\left(P_{n}(X, \beta), \chi_{P}\right), \\
N_{n, \beta}=\int_{\left[\mathcal{M}_{n}(X, \beta)\right]^{\text {vir }}} 1 & =e\left(\mathcal{M}_{n}(X, \beta), \chi_{\mathcal{M}}\right)
\end{aligned}
$$

are the weighted Euler characteristics.

The following property holds even though the map (1.2) may be neither smooth nor surjective. The result underpins the whole paper.

Theorem 4. $\chi_{P}=(-1)^{n-1} \phi_{n}^{*} \chi_{\mathcal{M}}$.

Proof. We work locally around one point $(F, s)$ of $P_{n}(X, \beta)$. By the irreducibility of $\beta$, the Cohen-Macaulay support $C$ of $F$ is reduced and irreducible. Hence, $C$ is generically nonsingular. There exists a local smooth divisor $D_{C}$ which intersects $C$ (and all nearby $C$ in the same homology class) transversally in a single point. We may also assume $D_{C} \cap C$ to be disjoint from the zeros of $s$.

Let $k \geq 0$. Tensoring with $\mathcal{O}\left(k D_{C}\right)$ and the canonical section $s_{D_{C}}^{k}$ yields a map of analytic open sets:

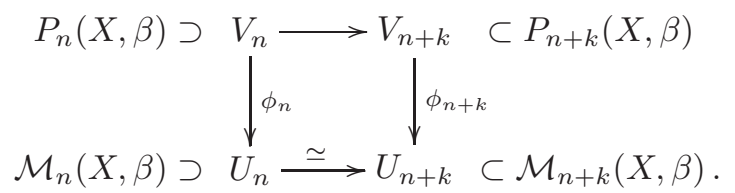

Here, $U_{n}$ is a sufficiently small analytic neighborhood of $F$. Since $\chi^{B}$-functions depend only on the local scheme structure [3], the bottom isomorphism makes the $\chi^{B}$-functions of the two-sheaf moduli spaces $\mathcal{M}$ locally the same. We call them $\chi_{\mathcal{M}}$. The open sets

$$
V_{n}=\phi_{n}^{-1}\left(U_{n}\right), \quad V_{n+k}=\phi_{n+k}^{-1}\left(U_{n+k}\right)
$$

contain $(F, s)$ and $\left(F\left(k D_{C}\right), s . s_{D_{C}}^{k}\right)$, respectively. For $k$ sufficiently large, $\phi_{n+k}$ is a smooth $\mathbb{P}^{n+k-1}$-bundle.

By making $V_{n+k}$ smaller if necessary, the map $V_{n} \rightarrow V_{n+k}$ admits a left inverse

$$
V_{n} \stackrel{\psi}{\leftarrow} V_{n+k}
$$

given by forgetting about the $k$ points close to $D_{C}$. The map $\psi$ is locally smooth with fibre the $k$ th symmetric product of an open subset of a nonsingular curve.

We calculate the $\chi^{B}$-function of $V_{n+k}$ in two different ways around the commutative diagram (1.6), using (1.5) applied to the two smooth maps $\psi$ and $\phi_{n+k}$. The two resulting expressions are

$$
(-1)^{n+k-1} \phi_{n+k}^{*} \chi_{\mathcal{M}}=(-1)^{k} \psi^{*} \chi_{P}
$$

Pulling back to $V_{n}$ gives

$$
(-1)^{n+k-1} \phi_{n}^{*} \chi_{\mathcal{M}}=(-1)^{k} \chi_{P}
$$

Multiplying by $(-1)^{k}$ gives the result.

\footnotetext{
${ }^{7}$ For convenience, we will often drop the superscripted $B$ in the notation.
} 


\section{BPS RATIONALITY}

2.1. Results. Let $X$ be a Calabi-Yau 3-fold and $\beta \in H_{2}(X, \mathbb{Z})$ an irreducible class. Let $g$ be the maximal arithmetic genus of a curve in the class $\beta$. Following the notation of Section 1, let

$$
P_{n, \beta}=\int_{\left[P_{n}(X, \beta)\right]} 1, \quad N_{n, \beta}=\int_{\left[\mathcal{M}_{n}(X, \beta)\right]^{v i r}} 1
$$

denote the invariants of [26, 31].

Proposition 2.1. For $\beta$ an irreducible class, $N_{1, \beta}=N_{n, \beta}$ for all $n$.

Proof. Let $F$ be a stable sheaf determining a moduli point of $\mathcal{M}_{1}(X, \beta)$. Let $C$ be the support of $F$. As in the proof of Theorem 4 let $D_{C}$ be a transverse divisor meeting $C$ in 1 point. Let $U_{1} \subset \mathcal{M}_{1}(X, \beta)$ be the analytic open set of sheaves supported on curves with a single transverse intersection with $D_{C}$. Tensoring with multiples of $\mathcal{O}\left(D_{C}\right)$ makes $U_{1}$ isomorphic to a corresponding open set $U_{n}$ in each $\mathcal{M}_{n}(X, \beta)$.

If $\mathcal{M}_{1}(X, \beta)$ is covered by finitely many open sets $U_{1}^{i}$ of the above form, the corresponding open sets $U_{n}^{i}$ cover $\mathcal{M}_{n}(X, \beta)$. By construction, the intersections

$$
U_{1}^{i} \cap U_{1}^{j}, \quad U_{1}^{i} \cap U_{1}^{j} \cap U_{1}^{k}, \ldots
$$

are isomorphic to the corresponding intersections

$$
U_{n}^{i} \cap U_{n}^{j}, \quad U_{n}^{i} \cap U_{n}^{j} \cap U_{n}^{k}, \ldots .
$$

Calculating the weighted Euler characteristics of the spaces $\mathcal{M}_{1}(X, \beta)$ and $\mathcal{M}_{n}(X, \beta)$ as a sum of weighted Euler characteristics of the $U^{i}$ (minus the weighted Euler characteristics of their intersections, plus the triple intersections and so on), we find that $N_{1, \beta}=N_{n, \beta}$.

Proposition 2.2. Let $\beta \in H_{2}(X, \mathbb{Z})$ be an irreducible class. The invariants $P_{n, \beta}$ satisfy the following identities:

$$
\begin{aligned}
P_{n, \beta}=(-1)^{n-1} n N_{1, \beta}, & & g \leq n, \\
P_{n, \beta}-P_{-n, \beta}=(-1)^{n-1} n N_{1, \beta}, & & -g<n<g . \\
P_{n, \beta}=0, & & n \leq-g .
\end{aligned}
$$

Proof. An element $(F, s)$ of $P_{n}(X, \beta)$ yields an exact sequence

$$
0 \rightarrow \mathcal{O}_{C} \stackrel{s}{\rightarrow} F \rightarrow Q \rightarrow 0,
$$

where $Q$ has 0 -dimensional support. We obtain the inequality

$$
n=\chi(F)=\chi\left(\mathcal{O}_{C}\right)+\chi(Q) \geq 1-g+0>-g .
$$

Therefore $P_{n}(X, \beta)$ is empty for $n \leq-g$, which implies (2.5). We verify (2.3) and (2.4) simultaneously by proving (2.4) for all $n \in \mathbb{Z}$.

If $F$ is a line bundle on a nonsingular curve $C \subset X$, then Serre duality relates $F$ and $F^{-1} \otimes K_{C}$. More generally, there is a map

$$
\begin{aligned}
\mathcal{M}_{n}(X, \beta) & \rightarrow \mathcal{M}_{-n}(X, \beta) \\
F & \mapsto \mathscr{E} x t_{X}^{2}\left(F, K_{X}\right) .
\end{aligned}
$$


Since $F$ is pure, $F$ has homological dimension 2 [12, Proposition 1.1.10], so $\mathscr{E} x t^{\geq 3}\left(F, K_{X}\right)=0$. Similarly $\mathscr{E} x t^{\leq 1}\left(F, K_{X}\right)=0$ because $F$ is supported in codimension 2. Therefore

$$
\mathscr{E} x t^{2}\left(F, K_{X}\right) \cong R \mathscr{H} o m\left(F, K_{X}\right)[2],
$$

which has the same Chern classes as elements of $\mathcal{M}_{-n}(X, \beta)$.

Pick a 3-term locally free resolution of $F$,

$$
0 \rightarrow F_{2} \rightarrow F_{1} \rightarrow F_{0} \rightarrow F \rightarrow 0 .
$$

Applying $\mathscr{H} o m\left(\cdot, K_{X}\right)$ gives a 3 -term locally free resolution

$$
0 \rightarrow F_{0}^{*} \otimes K_{X} \rightarrow F_{1}^{*} \otimes K_{X} \rightarrow F_{2}^{*} \otimes K_{X} \rightarrow \mathscr{E} x t^{2}\left(F, K_{X}\right) \rightarrow 0
$$

of $\mathscr{E} x t^{2}\left(F, K_{X}\right)$. Therefore by [12, Proposition 1.1.10] $\mathscr{E} x t^{2}\left(F, K_{X}\right)$ is a pure sheaf. By the irreducibility assumption, $\mathscr{E} x t^{2}\left(F, K_{X}\right)$ is stable and indeed defines an element of $\mathcal{M}_{-n}(X, \beta)$.

The map (2.6) is an involution and hence yields an isomorphism

$$
\mathcal{M}_{n}(X, \beta) \cong \mathcal{M}_{-n}(X, \beta) .
$$

We may therefore consider the projections $\phi_{n}$ and $\phi_{-n}$ (1.2) to fibre $P_{n}(X, \beta)$ and $P_{-n}(X, \beta)$ over the same space $\mathcal{M}_{n, \beta}$. We have

$$
H^{0}\left(\mathscr{E} x t^{2}\left(F, K_{X}\right)\right) \cong \operatorname{Ext}^{2}\left(F, K_{X}\right) \cong H^{1}(F)^{*}
$$

by Serre duality on $X$. The fibres of $\phi_{n}$ and $\phi_{-n}$ over $F \in \mathcal{M}_{n}(X, \beta)$ are therefore

$$
\mathbb{P}\left(H^{0}(F)\right) \text { and } \mathbb{P}\left(H^{1}(F)^{*}\right),
$$

respectively.

We stratify $\mathcal{M}_{n}(X, \beta)$ by the dimension of $H^{0}(F)$,

$$
\mathcal{M}_{n}(X, \beta)=\bigcup_{r} V_{r}
$$

where $V_{r}$ is the locus of sheaves $F$ with $h^{0}(F)=r$. There are induced stratifications $\phi_{ \pm n}^{-1}\left(V_{r}\right)$ of $P_{n}(X, \beta)$ and $P_{-n}(X, \beta)$. By [3], we may calculate the invariants $P_{ \pm n, \beta}$ via these stratifications as

$$
P_{ \pm n, \beta}=\sum_{r} e\left(\phi_{ \pm n}^{-1}\left(V_{r}\right),\left.\chi_{P}\right|_{\phi_{ \pm n}^{-1}\left(V_{r}\right)}\right)=\sum_{r}(-1)^{n-1} e\left(\phi_{ \pm n}^{-1}\left(V_{r}\right), \phi_{ \pm n}^{*} \chi_{\mathcal{M}}\right),
$$

with the last equality following from Theorem 4 . The $\chi^{B}$-function is the same constant on the fibres of both fibrations $\phi_{ \pm n}$.

By (2.7), over $V_{r}, \phi_{n}$ is a $\mathbb{P}^{r-1}$-bundle and $\phi_{-n}$ is a $\mathbb{P}^{r-n-1}$-bundle. These fibres have Euler characteristics $r$ and $r-n$, respectively. We find that

$$
P_{n, \beta}=\sum_{r}(-1)^{n-1} r e\left(V_{r}, \chi_{\mathcal{M}}\right)
$$

and

$$
P_{-n, \beta}=\sum_{r}(-1)^{n-1}(r-n) e\left(V_{r}, \chi_{\mathcal{M}}\right) .
$$

Subtracting gives

$$
\begin{aligned}
P_{n, \beta}-P_{-n, \beta} & =(-1)^{n-1} n \sum_{r} e\left(V_{r}, \chi_{\mathcal{M}}\right) \\
& =(-1)^{n-1} n e\left(\mathcal{M}_{n}(X, \beta), \chi_{\mathcal{M}}\right)=(-1)^{n-1} n N_{n, \beta} .
\end{aligned}
$$


By Proposition 2.2, the generating series

$$
Z_{\beta}(q)=\sum_{n} P_{n, \beta} q^{n}
$$

is the Laurent expansion of rational function in $q$, completing the proof of Theorem 1. However, a stronger statement can be made. Any Laurent series such as (2.8) can be written as

$$
Z_{\beta}(q)=\sum_{r} n_{r, \beta} q^{1-r}(1+q)^{2 r-2}
$$

where the sum is over all $r \in \mathbb{Z}$ and only finitely many terms with $r \geq 0$ are nonzero; see [26]. Moreover the integrality of the coefficients $P_{n, \beta}$ of (2.8) is equivalent to the integrality of the $n_{r, \beta}$.

The conditions (2.3) - 2.5) easily imply the vanishing of $n_{r, \beta}$ for $r<0$ and $r>g$. Therefore, by Proposition 2.2, $Z_{P, \beta}(q)$ can be written uniquely in the BPS form

$$
Z_{\beta}(q)=\sum_{r=0}^{g} n_{r, \beta} q^{1-r}(1+q)^{2 r-2}
$$

for integers $n_{r, \beta}$ which vanish for $r<0$ and for $r$ greater than the largest genus $g$ of a holomorphic curve in the class $\beta$. Since (2.10) is invariant under $q \leftrightarrow q^{-1}$, Theorems 2 and 3 are proven.

2.2. Remarks. From formula (2.10), we find that the genus 0 BPS invariant $n_{0, \beta}$ equals the DT invariant of sheaves $N_{1, \beta}$ in agreement with the proposal of S. Katz [15]. In fact, Katz expects

$$
n_{0, \beta}=N_{1, \beta}
$$

to hold in much greater generality.

Our sheaf-theoretic definition of BPS invariants 2.10 in the irreducible case is the first rigorous and manifestly deformation-invariant approach. Other papers on the subject [10, 30, 32] have defined BPS invariants following the original perspective of [7, 8] using $\left(\mathfrak{s l}_{2} \times \mathfrak{s l}_{2}\right)$-actions on sophisticated cohomology theories, but have been unable to incorporate the virtual class. These definitions are therefore unlikely to be deformation invariant. Our definition is rather simpler, and more in line with the viewpoint of [16].

It should be possible to extend our results to the Fano case for any class $\beta$. After imposing the requisite number of incidence conditions to cut the virtual dimension to 0 , the Fano case behaves like the Calabi-Yau case for irreducible $\beta$, as all other invariants vanish. However, at present, the analogue of $\chi^{B}$ is missing in the Fano case.

2.3. Wall-crossing. Arend Bayer [1] and Yukinoba Toda 33 . have made the beautiful observation that (2.4) should be seen as a wall-crossing formula. In fact, the wall-crossing is much simpler than the wall-crossing conjectured in 26] to equate the invariants $P_{n, \beta}$ to the reduced DT invariants of [23]. For any

$$
I^{\bullet}=\left\{\mathcal{O}_{X} \rightarrow F\right\} \in P_{n}(X, \beta),
$$

we have the obvious exact triangle

$$
F[-1] \rightarrow I^{\bullet} \rightarrow \mathcal{O}_{X}
$$


Taking the derived dual gives

$$
\mathcal{O}_{X} \rightarrow\left(I^{\bullet}\right)^{\vee} \rightarrow \mathscr{E} x t^{2}\left(F, K_{X}\right)[-1]
$$

where $\mathscr{E} x t^{2}\left(F, K_{X}\right) \in \mathcal{M}_{-n}(X, \beta)$ is the sheaf dual to $F$ under the duality (2.6).

Start with a stability condition for which the complexes $I^{\bullet} \in P_{n, \beta}$ and the sheaves $F, \mathcal{O}_{X}$ are stable. In particular, the phase of $F[-1]$ should be less than that of $\mathcal{O}_{X}$ due to the exact triangle (2.11). Now pass through a codimension 1 wall in the space of stability conditions so that the phase of $F[-1]$ crosses that of $\mathcal{O}_{X}$. The extensions (2.11) become unstable, while extensions in the opposite direction (2.12) become stable. Therefore, on the other side of the wall, the stable objects are the derived duals of the complexes made out of stable pairs in $P_{-n}(X, \beta)$.

Ideally, wall-crossing should be studied with Bridgeland stability conditions. However, at present, their existence is conjectural. If instead we use Bayer's polynomial stability conditions or Toda's limit stability conditions, then the analysis can be made precise. These stability conditions have been constructed, and the stable objects are as claimed above [1, 33.

Since the pieces $\mathcal{O}_{X}, F, \mathscr{E} x t^{2}\left(F, K_{X}\right)$ occurring in the complexes are also stable in these stability conditions, Joyce's conjectural wall-crossing formula 14 takes a very simple form. We count only complexes of trivial determinant throughout. The invariant counting the stable objects $I^{\bullet} \in P_{n}(X, \beta)$ on one side of the wall should differ from those $\left(I^{\bullet}\right)^{\vee} \in P_{-n}(X, \beta)$ on the other side by

$$
(-1)^{\chi\left(\mathcal{O}_{X}, F[-1]\right)} \chi\left(\mathcal{O}_{X}, F[-1]\right) \cdot \#\left(\mathcal{O}_{X}\right) \cdot \#(F),
$$

where

$$
\chi\left(\mathcal{O}_{X}, F[-1]\right)=\sum_{i}(-1)^{i} \operatorname{dim} \operatorname{Ext}^{i}\left(\mathcal{O}_{X}, F[-1]\right)=-n,
$$

and \# denotes the virtual number of elements of the moduli space of stable objects of the corresponding type. For us, 2.13) predicts that

$$
P_{n, \beta}-P_{-n, \beta}=(-1)^{-n}(-n) \cdot 1 \cdot N_{n, \beta}
$$

in precise agreement with (2.4). Perhaps (2.14) is the first nontrivial example of a wall-crossing formula in the derived category that can be rigorously proved.

Toda 33 has gone further with wall-crossings for arbitrary (rather than irreducible) stable pairs. Using the work of Joyce [14, he proves analogues of Theorems 1 and 2 for the Euler characteristics of the moduli spaces of stable pairs. Once Behrend's function $\chi^{B}$ and the identities of Kontsevich-Soibelman about the value $\chi^{B}$ on extensions 19. have been incorporated into Joyce's work, Theorems 1 and 2 for all classes on Calabi-Yau 3-folds should be obtained.

\section{LOCAL DEFINITION OF BPS INVARIANTS}

3.1. Fixed curve. Let $X$ be a Calabi-Yau 3-fold. Throughout this section we fix a Cohen-Macaulay curve $C \subset X$ in the irreducible class $\beta$ of arithmetic genus $g=1-\chi\left(\mathcal{O}_{C}\right)$. The curve $C$ is reduced and irreducible. Let

$$
P_{n}(C) \subset P_{n}(X, \beta) \text { and } \mathcal{M}_{n}(C) \subset \mathcal{M}_{n}(X, \beta)
$$


denote the loci of stable pairs and pure sheaves supported on $C$. Define localised invariant 8 by

$$
P_{n, C}=e\left(P_{n}(X, C),\left.\chi_{P}\right|_{P_{n}(C)}\right) .
$$

In Proposition 2.2, we computed the weighted Euler characteristics of the spaces $P_{n}(X, \beta)$ using the map (1.2) to $\mathcal{M}_{n}(X, \beta)$. We can instead restrict attention to the loci $P_{n}(C) \subset P_{n}(X, \beta)$, the inverse images of $\mathcal{M}_{n}(C) \subset \mathcal{M}_{n}(X, \beta)$. The same proof applies, since $\bigcup_{n} \mathcal{M}_{n}(C)$ is invariant under tensoring by line bundles and under the duality (2.6). We therefore obtain the same identities for localised invariants:

$$
\begin{aligned}
P_{n, C} & =(-1)^{n-1} n N_{1, C}, & & g \leq n, \\
P_{n, C}-P_{-n, C} & =(-1)^{n-1} n N_{1, C}, & & -g<n<g, \\
P_{n, C} & =0, & & n \leq-g,
\end{aligned}
$$

where $N_{1, C}=e\left(\mathcal{M}_{n}(C),\left.\chi_{\mathcal{M}}\right|_{\mathcal{M}_{n}(C)}\right)$. Thus, the generating series

$$
Z_{C}(q)=\sum_{n} P_{n, C} q^{n}
$$

can be written uniquely as

$$
Z_{C}(q)=\sum_{r=0}^{g} n_{r, C} q^{1-r}(1+q)^{2 r-2}
$$

for integers $n_{r, C}, r=0, \ldots, g$.

3.2. Chow. Let $\operatorname{Chow}(X, \beta)$ denote the variety of 1 -dimensional cycles in the class $\beta$. Since $\beta$ is irreducible, the cycles have no multiplicities. In fact, $\operatorname{Chow}(X, \beta)$ parameterises Cohen-Macaulay curves in class $\beta$.

The spaces $P_{n}(X, \beta)$ and $\mathcal{M}_{n}(X, \beta)$ map to $\operatorname{Chow}(X, \beta)$ with fibres $P_{n}(C)$ and $\mathcal{M}_{n}(C)$, respectively. We may calculate weighted Euler characteristics of $P_{n}(X, \beta)$ and $\mathcal{M}_{n}(X, \beta)$ as weighted Euler characteristics of $\operatorname{Chow}(X, \beta)$, with weight function the weighted Euler characteristics of the fibres. More precisely, the integers $P_{n, C}$ (3.1) define constructible functions

$$
\begin{aligned}
\operatorname{Chow}(X, \beta) & \rightarrow \mathbb{Z}, \\
C & \mapsto P_{n, C},
\end{aligned}
$$

whose weighted Euler characteristics are the integers $P_{n, \beta}$. Similarly, $Z_{C}(q)$ (3.2) defines a $\mathbb{Z}((q))$-valued constructible function on $\operatorname{Chow}(X, \beta)$ with weighted Euler characteristic $Z_{\beta}(q)$.

Therefore the $n_{r, C}$ (3.3) define constructible functions

$$
\begin{aligned}
\tilde{n}_{r, \beta}: \operatorname{Chow}(X, \beta) & \rightarrow \mathbb{Z}, \\
C & \mapsto n_{r, C},
\end{aligned}
$$

such that the BPS invariants $n_{r, \beta}$ of (2.10) are the weighted Euler characteristics

$$
n_{r, \beta}=e\left(\operatorname{Chow}(X, \beta), \tilde{n}_{r, \beta}\right) .
$$

We call $\tilde{n}_{r, \beta}(C)=n_{r, C}$ the contribution of $C \subset X$ to $n_{r, \beta}$.

${ }^{8}$ These are not invariants of $C$ alone. The dependence on the embedding $C \subset X$ comes through $\chi_{P}$. 
Since these definitions hide behind a lot of formulae, their exact meaning is rather opaque. We would like to be able to compute the contributions $n_{r, C}$ directly, without computing all of the invariants $P_{n, C}$ of the stable pairs. In fact, the invariants $n_{r, \beta}$ are the more fundamental invariants, from which all others $(\mathrm{GW}$, DT, stable pairs) should follow. Naively, we expect [7, 8, 16] that within the class $\beta$, the invariant $n_{r, \beta}$ counts curves of geometric genus $r$ "in $X$ ". Here "in" is to be interpreted loosely, including, as we discover below, maps which are merely generically embeddings. In particular, a nonsingular curve

$$
C \subset X
$$

of genus $g$ should contribute only to $n_{g, \beta}$, while a reduced irreducible curve $C \subset X$ of arithmetic genus $g$ and geometric genus $h<g$ should contribute to at most $n_{h}, n_{h+1}, \ldots, n_{g}$.

3.3. Nonsingular curves. The BPS contributions $n_{r, C}$ of a nonsingular curve $C$ are easy to compute. First, we need to understand the local deformation theory of the pairs' spaces $P_{n}(X, \beta)$ about the locus $P_{n}(C)$ of pairs supported on $C$. The answer turns out to be very simple with all of the $\chi^{B}$-functions $\chi_{P}$ of these spaces being equal, up to sign, to the same constant $\chi_{\mathcal{M}}\left(\mathcal{O}_{C}\right)$. Here, as before, $\chi_{\mathcal{M}}$ is the $\chi$-function of the moduli space

$$
\mathcal{M}_{1-g}(X, \beta) \ni \mathcal{O}_{C}
$$

of sheaves of the Hilbert polynomial $k \mapsto k \int_{\beta} c_{1}(L)+(1-g)$.

Lemma 3.4. $\left.\chi_{P}\right|_{P_{n}(C)}=(-1)^{n-1} \chi_{\mathcal{M}}\left(\mathcal{O}_{C}\right)$ is constant.

Proof. We follow the proof of Theorem 4, As there, in a neighbourhood of the locus

$$
\mathcal{M}_{1-g}(C) \subset \mathcal{M}_{n}(X, \beta)
$$

of sheaves supported on $C, \mathcal{M}_{1-g}(X, \beta)$ is isomorphic to all other such moduli spaces

$$
\mathcal{M}_{1-g+i}(X, \beta) \ni \mathcal{O}_{C}(D)
$$

where $D$ is a divisor on $C$ of degree $i$. We use the same argument as before: extend $D$ to a local divisor in $X$ (using the nonsingularity of $C$ ) and map

$$
\mathcal{M}_{1-g}(X, \beta) \stackrel{\otimes \mathcal{O}(D)}{\longrightarrow} \mathcal{M}_{1-g+i}(X, \beta)
$$

taking $\mathcal{O}_{C}$ to $\mathcal{O}_{C}(D)$. Thus

$$
\chi_{\mathcal{M}}\left(\mathcal{O}_{C}\right)=\chi_{\mathcal{M}}\left(\mathcal{O}_{C}(D)\right)
$$

and the $\chi^{B}$-function is identically constant over the loci of sheaves supported on $C$, since $D$ is arbitrary. By Theorem 4 the $\chi^{B}$-functions of all of the moduli spaces $P_{1-g+i}(X, \beta)$ of pairs take the constant value $(-1)^{-g+i} \chi_{\mathcal{M}}\left(\mathcal{O}_{C}\right)$ on restriction to $P_{1-g+i}(C)$.

The space of pairs $P_{1-g+i}(C)$ supported on $C$ is the $i$ th symmetric product $S^{i} C$ of $C$ (a proof of a more general fact is given in Proposition B.5 below). By Lemma 3.4. the invariants of the local stable pairs are

$$
P_{1-g+i, C}=(-1)^{-g+i} \chi_{\mathcal{M}}\left(\mathcal{O}_{C}\right) e\left(S^{i} C\right) .
$$


In this case the interpretation of the formula (3.3) is clear. For any manifold $M$, the generating function for the numbers $(-1)^{i} e\left(S^{i} M\right)$ is

$$
(1+q)^{-e(M)}=1-e(M) q+\frac{e(M)(e(M)+1)}{2} q^{2}-\cdots .
$$

Therefore $Z_{C}(q)$, the contribution of $C$ to $Z_{P, \beta}(q)$, is

$$
\begin{aligned}
(-1)^{g} \chi_{\mathcal{M}}\left(\mathcal{O}_{C}\right)\left(q^{1-g}-e(C) q^{2-g}+e\left(S^{2} C\right) q^{3-g}-e\left(S^{3} C\right) q^{4-g}+\cdots\right) \\
=(-1)^{g} \chi_{\mathcal{M}}\left(\mathcal{O}_{C}\right) q^{1-g}(1+q)^{2 g-2} .
\end{aligned}
$$

Since $q^{1-g}(1+q)^{2 g-2}$ is precisely the contribution of $n_{g, C}$ to (3.3), we have proved the following.

Proposition 3.6. A nonsingular curve $C \subset X$ of genus $g$ contributes

$$
n_{g, C}=(-1)^{g} \chi_{\mathcal{M}}\left(\mathcal{O}_{C}\right)
$$

to $n_{g, \beta}$, and $n_{r, C}=0$ for $r \neq g$.

3.4. Singular curves: Discussion. For smooth curves, the geometry of the formulae (3.3) is very simple. Remarkably, the BPS formalism makes sense in the singular case also. To start, we expand the formulae (3.3) and read off the $n_{r, C}$ inductively:

$$
\begin{aligned}
& P_{1-g, C}=n_{g, C}, \\
& P_{2-g, C}=-e\left(\Sigma_{g}\right) n_{g, C}+n_{g-1, C}, \\
& P_{3-g, C}=e\left(S^{2} \Sigma_{g}\right) n_{g, C}-e\left(\Sigma_{g-1}\right) n_{g-1, C}+n_{g-2, C},
\end{aligned}
$$

and so on. Here and below we denote a smooth compact 2-manifold of genus $g$ by $\Sigma_{g}$.

The formulae (3.7) tell us, inductively, what $C$ contributes to each BPS number. The moduli space $P_{1-g}(C)$ consists of the single point $\left(\mathcal{O}_{C}, 1\right)$. By Theorem 4 $P_{1-g, C}=(-1)^{g} \chi_{\mathcal{M}}\left(\mathcal{O}_{C}\right)$, so by (3.7),

$$
n_{g, C}=(-1)^{g} \chi_{\mathcal{M}}\left(\mathcal{O}_{C}\right) \text {. }
$$

The contribution of the $n_{g, C}$ term to $P_{2-g, C}$ is then

$$
-(-1)^{g} e\left(\Sigma_{g}\right) \chi_{\mathcal{M}}\left(\mathcal{O}_{C}\right) \text {. }
$$

If $C$ is nonsingular, (3.8) is precisely the contribution of the space of pairs $P_{2-g}(C) \cong$ $C$ supported on $C$, but for $C$ singular, $P_{2-g, C}$ is the more complicated weighted Euler characteristic

$$
e\left(P_{2-g}(C),\left.\chi_{P}\right|_{P_{2-g}(C)}\right) .
$$

We define $n_{g-1, C}$ to be the discrepancy between these two Euler characteristics:

$$
n_{g-1, C}=P_{2-g, C}+(-1)^{g} e\left(\Sigma_{g}\right) \chi_{\mathcal{M}}\left(\mathcal{O}_{C}\right) .
$$

For example, consider a curve $C \subset X$ with 1 node for which the moduli space of sheaves $\mathcal{M}_{1-g}(X, \beta)$ is nonsingular in a neighbourhood of $\mathcal{M}_{1-g}(C)$. Let \pm denote the sign $(-1)^{g+\operatorname{dim} \mathcal{M}_{1-g}(X, \beta)}$. Then $n_{g, C}= \pm 1, P_{2-g}(C) \cong C$, and so

$$
n_{g-1, C}= \pm\left(-e(C)+e\left(\Sigma_{g}\right)\right)=\mp 1 .
$$

We proceed inductively by viewing $C$ as contributing $n_{g, C}$ nonsingular curves of genus $g$ and $n_{g-1, C}$ nonsingular curves of genus $g-1$. These genus $g$ and $g-1$ curves contribute

$$
e\left(S^{2} \Sigma_{g}\right) n_{g, C}-e\left(\Sigma_{g-1}\right) n_{g-1, C}
$$


to $P_{3-g, \beta}$, as in (3.7). The discrepancy

$$
n_{g-2, C}=P_{3-g, C}-e\left(S^{2} \Sigma_{g}\right) n_{g, C}+e\left(\Sigma_{g-1}\right) n_{g-1, C}
$$

is what we define to be the number of genus $g-2$ curves supported on $C \subset X$.

These formulae quickly become unmanageable, which is why we use the more concise generating functions (3.3) to which they are equivalent. A number of miraculous cancellations of Euler characteristics and $\chi^{B}$-functions must occur for a singular curve of geometric genus $h$ to have $n_{r, C}=0$ for $r<h$. We will obtain these cancellations from an interplay between Serre duality and Theorem 4 .

3.5. Singular curves: Results. Let $\operatorname{Pic}(C)$ denote the group of line bundles of degree 0 on $C$. There is an action of $\operatorname{Pic}(C)$ on $\mathcal{M}_{n}(C)$ by tensoring. Let

$$
\left\{O_{i} \subset \mathcal{M}_{n}(C)\right\}_{i \in I}
$$

be the set of $\operatorname{Pic}(C)$-orbits. The index set $I$ need not be finite. By convention we fix $O_{0}$ to be the orbit consisting of line bundles of degree $n-1+g$ on $C$.

Fix a local effective divisor $D_{C}$ intersecting $C$ transversely at a nonsingular point. Tensoring with multiples of $\mathcal{O}\left(D_{C}\right)$ fixes local isomorphisms between all of the $\mathcal{M}_{n}(X, \beta)$ in a neighbourhood of $\mathcal{M}_{n}(C)$. These isomorphisms preserve the orbit types $O_{i}$. Therefore, we think of the $\mathcal{M}_{n}(X, \beta)$ and their subloci $O_{i}$ as (locally) independent of $n$.

The duality (2.6) maps every orbit $O_{i} \subset \mathcal{M}_{n}(C)$ to another orbit $O_{i^{\prime}} \subset \mathcal{M}_{-n}(C)$ since

$$
\mathscr{E} x t^{2}\left(F \otimes L, K_{X}\right) \cong L^{-1} \otimes \mathscr{E} x t^{2}\left(F, K_{X}\right)
$$

for any (local) line bundle $L$. Hence, we obtain an involution

$$
i \leftrightarrow i^{\prime}
$$

on the indexing set $I$.

Lemma 3.11. The restriction $\left.\chi_{\mathcal{M}}\right|_{O_{i}}$ of the $\chi^{B}$-function of $\mathcal{M}_{n}(X, \beta)$ is a constant $\chi_{i}$ on each orbit $O_{i}$ (independent of $n$ ). Moreover $\chi_{i}=\chi_{i^{\prime}}$.

Proof. Every line bundle $L \in \operatorname{Pic}(C)$ can be trivialised over the finite singular set of $C$, so is linearly equivalent to a (noneffective) divisor supported on the nonsingular locus of $C$. The latter can be extended to a local divisor in $X$. Therefore $L$ is the restriction of a line bundle $L$ defined on a neighbourhood of $C \subset X$. Then, the map

$$
\mathcal{M}_{n}(X, \beta) \stackrel{\otimes L}{\longrightarrow} \mathcal{M}_{n}(X, \beta),
$$

defined only locally in a neighbourhood of $\mathcal{M}_{n}(C)$, is a local isomorphism and so preserves $\chi^{B}$-functions. Thus,

$$
\chi_{\mathcal{M}}(F)=\chi_{\mathcal{M}}(F \otimes L)
$$

for any $F \in \mathcal{M}_{n}(C) \subset \mathcal{M}_{n}(X, \beta)$ and $L \in \operatorname{Pic}(C)$.

Since the construction commutes with the isomorphisms

$$
\mathcal{M}_{n}(C) \rightarrow \mathcal{M}_{n+1}(C)
$$

given by tensoring with $\mathcal{O}\left(D_{C}\right), \chi_{i}$ is independent of $n$. Since the duality (2.6) preserves $\chi^{B}$-functions, $\chi_{i}=\chi_{i^{\prime}}$. 
Let $Z \subset C$ be the support of the singularities of $C$, and let

$$
C^{0}=C \backslash Z
$$

be the nonsingular locus. Let

$$
\mu_{Z}=(2-2 g)-e\left(C^{0}\right)
$$

be the Euler characteristic of the Milnor fibre of $Z$ : the sum over the components of $Z$ of one minus the Milnor number of the component. The invariant $\mu_{Z}$ depends only on the analytic germ of $Z \subset C$. Define

$$
Q_{n}(C, Z) \subset P_{1-g+n}(C)
$$

to be the locus of pairs whose cokernel $Q$ (which has length $n$ ) is supported entirely at $Z$.

Define an admissible subset

$$
J=\bigcup_{n} J_{n} \subset \bigcup_{n} \mathcal{M}_{n}(C)
$$

to be a constructible subset invariant under tensoring with line bundles on $C$ of any degree, and invariant under the duality (2.6). Equivalently $J$ is a (possibly infinite) union of orbit pairs $O_{i} \cup O_{i^{\prime}}$ (if $i=i^{\prime}$ is fixed by the duality (3.10), then this is just $O_{i}$ ) and their translations by line bundles of nonzero degree.

We set $Q_{n}(C, Z)_{J}$ to be the locus of points in $Q_{n}(C, Z)$ whose underlying sheaf lies in $J$.

Proposition 3.13. For any admissible $J$, the generating function of signed topological Euler characteristics

$$
\sum_{n=0}^{\infty}(-1)^{n} e\left(Q_{n}(C, Z)_{J}\right) q^{n}
$$

can be written as

$$
\sum_{r=0}^{g} n_{r}(J) q^{g-r}(1+q)^{2 r-2 g-\mu_{Z}}
$$

for integers $n_{r}(J), r=0, \ldots, g$.

Proof. We first use the same argument as in Proposition 2.2 applied to the Euler characteristics of the fibres of the map $\phi_{n}$ from

$$
P_{n}(C)_{J}=\phi_{n}^{-1}(J)
$$

to $J_{n}$. The fibres are empty for $n \leq-g$.

The fibre $\mathbb{P}\left(H^{0}(F)\right)$ over $F \in \mathcal{M}_{n}(C)$ has Euler characteristic $h^{0}(F)$ while the fibre $\mathbb{P}\left(H^{1}(F)^{*}\right)$ over the dual

$$
\mathscr{E} x t_{X}^{2}\left(F, K_{X}\right) \in \mathcal{M}_{-n}(C)
$$

has Euler characteristic $h^{1}(F)$. If $F \in J_{n}$, then $\mathscr{E} x t^{2}\left(F, K_{X}\right) \in J_{-n}$. Since the difference between these two Euler characteristics is $n$, we obtain

$$
e_{n}-e_{-n}=(-1)^{n+g-1} n e\left(J_{1}\right), \quad \text { and } \quad e_{n}=0, \quad n \leq-g,
$$

where we have defined

$$
e_{n}=(-1)^{n+g-1} e\left(P_{n}(C)_{J}\right)
$$


and used $J_{n} \cong J_{1}$ for all $n$. Hence, we can write

$$
\sum_{n} e_{n} q^{n}=\sum_{r=0}^{g} n_{r}(J) q^{1-r}(1+q)^{2 r-2}
$$

uniquely with integers $n_{r}(J), r=0, \ldots, g$, with $n_{0}(J)=(-1)^{g} e\left(J_{1}\right)$.

Restricted to the nonsingular locus $C^{0}$, every stable pair corresponds to a nonzero section of a line bundle. The orbit type of the underlying sheaf is determined by its restriction to the germ of $Z \subset C$. Therefore, stratifying $P_{n}(C)_{J}$ by the length of that part of the cokernel $Q=F / \mathcal{O}_{C}$ of the pair supported on $Z$, we obtain the decomposition

$$
P_{n}(C)_{J}=\coprod_{k=0}^{n+g-1} Q_{k}(C, Z)_{J} \times S^{n+g-1-k}\left(C^{0}\right) .
$$

Taking generating series of signed Euler characteristics gives, by (3.5),

$$
\sum_{n} e_{n} q^{n}=\sum_{k=0}^{\infty}(-1)^{k} e\left(Q_{k}(C, Z)_{J}\right) q^{k+1-g}(1+q)^{-e\left(C^{0}\right)} .
$$

Substituting (3.14) gives

$$
\sum_{r=0}^{g} n_{r}(J) q^{1-r}(1+q)^{2 r-2}=q^{1-g}(1+q)^{2 g-2+\mu_{Z}} \sum_{k=0}^{\infty}(-1)^{k} e\left(Q_{k}(C, Z)_{J}\right) q^{k} .
$$

Rearranging implies the result.

The advantage of the spaces $Q_{k}(C, Z)$ is that they depend only on the germ of $Z \subset C$ (in the analytic topology) and not on $C$ or the genus $g$.

Lemma 3.16. Any curve with the same singularity germ as $Z \subset C$ has orbits $O_{i}, i \in I$ and admissible subsets $J$ in 1-1 correspondence with those of $C$. Moreover, the spaces $Q_{k}(C, Z)$ and $Q_{k}(C, Z)_{J}$ and the duality $i \leftrightarrow i^{\prime}$ depend only on the germ of $Z \subset C$.

Proof. Stable pairs supported on $C$ with given cokernel $Q$ are determined by the extension

$$
0 \rightarrow \mathcal{O}_{C} \rightarrow F \rightarrow Q \rightarrow 0
$$

whose class lies in

$$
\operatorname{Ext}_{C}^{1}\left(Q, \mathcal{O}_{C}\right)=H^{0}\left(\mathscr{E} x t_{C}^{1}\left(Q, \mathcal{O}_{C}\right)\right)
$$

The right side is just $\mathscr{E} x t_{C}^{1}\left(Q, \mathcal{O}_{C}\right)$, thought of as a $\mathbb{C}$-module. Thus the stable pair is determined by data entirely local to the support of $Q$.

Since the pairs $Q_{k}(C, Z) \subset P_{1-g+k}(C)$ have cokernel supported entirely at $Z$, they are determined by the germ of $Z \subset C$.

The sheaf $F$ underlying a stable pair is a line bundle on $C^{0}$. Hence, the orbit type is determined by the restriction of $F$ to the germ of $Z \subset C$. Similarly we claim that the action of the duality (2.6) on orbits $O_{i}$ is determined locally about $Z \subset C$ by

$$
F \mapsto \mathscr{H}_{C}\left(F, \omega_{C}\right)
$$

Here, we consider $F$ as an $\mathcal{O}_{C}$-module and use relative Serre duality for the embedding $\iota: C \hookrightarrow X$ :

$$
R \mathscr{H} \mathrm{m}_{X}\left(\iota_{*} F, K_{X}\right) \cong \iota_{*} R \mathscr{H}_{\mathrm{om}}\left(F, \omega_{C}\right)[-2] .
$$


Taking $h^{2}$ of both sides and using the exactness of $\iota_{*}$ gives

$$
\mathscr{E} x t_{X}^{2}\left(\iota_{*} F, K_{X}\right) \cong \iota_{*} \mathscr{H} o m_{C}\left(F, \omega_{C}\right),
$$

as required.

Hence, the admissible subsets $J$ are also determined by the germ of $Z \subset C$.

We can now compare $C$ to a curve of minimal genus with the same singularities. We let $g=g(C)$ denote the arithmetic genus of $C$, and $g(\bar{C})$ its geometric genus, i.e., the genus of the normalisation

$$
p: \bar{C} \rightarrow C .
$$

The $\delta$-invariant of $Z$ is the difference:

$$
\delta(Z)=g(C)-g(\bar{C})=\text { length }\left(\left(p_{*} \mathcal{O}_{\bar{C}}\right) / \mathcal{O}_{C}\right) .
$$

The $\delta$-invariant depends only on the germ of $Z \subset C$ (since $\left(p_{*} \mathcal{O}_{\bar{C}}\right) / \mathcal{O}_{C}$ is supported at $Z$ ). It is the minimal arithmetic genus of a curve $C^{\prime}$ containing $Z$ as a singularity: the arithmetic genus of a rational curve with singularity $Z$. Such curves exist in Calabi-Yau 3-folds.

Lemma 3.17. Given a germ of a singularity $Z \subset C$ of a space curve $C$, there exists a smooth projective 3 -fold $X^{\prime}$ containing a compact rational curve $C^{\prime}$ with singularities $Z^{\prime}$ such that the germ of $Z^{\prime} \subset C^{\prime}$ is isomorphic to the germ of $Z \subset C$. We may also arrange that the canonical bundle $K_{X^{\prime}}$ is trivial on all curves close to $C^{\prime}$.

Proof. Normalise $C$ at $Z$,

$$
p: \bar{C} \rightarrow C,
$$

and take an analytic neighbourhood of $p^{-1}(Z)$ consisting of a finite union of discs. Embed these discs into $\mathbb{P}^{1}$ and reapply $p$ to them to give a rational curve $C^{\prime}$ with singularities exactly $Z$.

The curve $C^{\prime}$ can be embedded in $\mathbb{P}^{N}$ and then mapped to $\mathbb{P}^{3}$ by a generic projection. Since the Zariski tangent spaces all have dimension $\leq 3$, the map $C^{\prime} \rightarrow \mathbb{P}^{3}$ will still be an embedding.

Fix a nonsingular divisor $D \subset \mathbb{P}^{3}$ of degree 2 which intersects $C^{\prime}$ transversally away from $Z$. Blow up $D \cap C^{\prime}$ to give a 3 -fold $X^{\prime}$ with exceptional divisor $E$. The proper transform $\bar{C}^{\prime} \cong C^{\prime}$ is a rational curve in $X^{\prime}$.

All nearby curves are also the proper transforms $\bar{C}^{\prime \prime}$ of curves

$$
C^{\prime \prime} \subset \mathbb{P}^{3}
$$

which pass through the points $D \cap C^{\prime}$. For all such curves, $\left.K_{\mathbb{P}^{3}}\right|_{C^{\prime \prime}}$ is isomorphic to the divisor $-\left(2 D \cap C^{\prime}\right) \subset C^{\prime \prime}$. Therefore,

$$
\left.\left.K_{X^{\prime}}\right|_{\bar{C}^{\prime \prime}} \cong K_{\mathbb{P}^{3}}\right|_{C^{\prime \prime}}+2 E \cap C^{\prime \prime}=-2 D \cap C^{\prime}+2 D \cap C^{\prime}
$$

is trivial.

Instead of studying $Q_{k}(C, Z)$ through $C$ of genus $g$, we can study the same space $Q_{k}\left(C^{\prime}, Z^{\prime}\right)$ (by Lemma 3.16) through $C^{\prime}$ of minimal arithmetic genus $\delta(Z)$. By Proposition 3.13, we obtain a stronger result:

$$
\sum_{n=0}^{\infty}(-1)^{n} e\left(Q_{n}(C, Z)_{J}\right) q^{n}=\sum_{r=0}^{\delta(Z)} n_{r}(J) q^{\delta(Z)-r}(1+q)^{2 r-2 \delta(Z)-\mu_{Z}} .
$$


Theorem 5. The curve $C$ contributes only to the BPS numbers $n_{r}$ for $r$ between the geometric and arithmetic genera of $C$ :

$$
n_{r, C}=0 \quad \text { for } r<g(\bar{C}) \text { or } r>g(C) .
$$

Proof. For each $j \in \mathbb{Z}$ set

$$
J(j)=\bigcup_{n} J(j)_{n} \subset \bigcup_{n} \mathcal{M}_{n}(C)
$$

to be the locus where the $\chi^{B}$-function $\chi_{\mathcal{M}}$ restricted from $\mathcal{M}_{n}(X, \beta)$ equals $j$. By Lemma 3.11, $J(j)$ is invariant under tensoring with line bundles and under the duality (2.6). Hence, $J(j)$ is admissible (3.12), being a (possibly infinite) union of orbit pairs $O_{i} \cup O_{i^{\prime}}$ and their translates by line bundles of nonzero degree.

By (3.15) we have the stratification

$$
P_{n}(C)_{J(j)}=\coprod_{k=0}^{n+g-1} Q_{k}(C, Z)_{J(j)} \times S^{n+g-1-k}\left(C^{0}\right),
$$

where, by Lemma 3.16 the loci $Q_{k}(C, Z)_{J(j)}$ depend only on the germ of $Z \subset C$. By Theorem 4 and (3.5),$P_{n}(C)_{J(j)}$ contributes

$$
\sum_{k=0}^{\infty}(-1)^{k-g} j e\left(Q_{k}(C, Z)_{J(j)}\right) q^{k+1-g}(1+q)^{-e\left(C^{0}\right)}
$$

to $Z_{C}(q)$. By (3.18), the above equals

$$
(-1)^{g} j \sum_{r=0}^{\delta(Z)} n_{r}(J(j)) q^{\delta(Z)-r+1-g}(1+q)^{2 r-2 \delta(Z)-\mu_{Z}-e\left(C^{0}\right)} .
$$

Setting $s=r+g(\bar{C})$, we rewrite the contribution as

$$
(-1)^{g} j \sum_{s=g(\bar{C})}^{g} n_{s-g(\bar{C})}(J(j)) q^{1-s}(1+q)^{2 s-2} .
$$

Since $\chi_{\mathcal{M}}$ takes only finitely many values, we add up over finitely many $J(j)$ to get

$$
Z_{C}(q)=\sum_{s=g(\bar{C})}^{g} n_{s, C} q^{1-s}(1+q)^{2 s-2},
$$

where $n_{s, C}=(-1)^{g} \sum_{j} j n_{s-g(\bar{C})}(J(j))$. We have obtained the required BPS form, with nonzero BPS numbers $n_{s, C}$ only for $g(\bar{C}) \leq s \leq g$.

3.6. Nodal curves. We illustrate the results of Section 3.5 in the case of nodal curves, where the formulae are rather simpler.

Consider first an irreducible elliptic curve $C$ with one node and normalisation

$$
\mathbb{P}^{1} \cong \bar{C} \stackrel{p}{\longrightarrow} C .
$$

The moduli space of sheaves $\mathcal{M}_{1}(C)$ is just a copy of $C$. There are two orbits $O_{i}$ of $\operatorname{Pic}(C): O_{0}$ is the smooth part $C^{0}$ of $C$ corresponding to degree one line bundles on $C$, and $O_{1}$ is the nodal point $Z$ corresponding to the pure sheaf $p_{*} \mathcal{O}_{\bar{C}}$.

The corresponding constant $\chi_{\mathcal{M}}$-functions are

$$
\chi_{0}=\chi_{\mathcal{M}}\left(\mathcal{O}_{C}\right), \quad \chi_{1}=\chi_{\mathcal{M}}\left(p_{*} \mathcal{O}_{\bar{C}}\right) .
$$


As we have seen,

$$
n_{1, C}=(-1)^{g} \chi_{0}=-\chi_{0} .
$$

Already for $n=1$ we have cohomology vanishing and $P_{1}(C)$ is a $\mathbb{P}^{0}$-bundle over $\mathcal{M}_{1}(C) \cong C$. Therefore,

$$
P_{1, C}=\chi_{0} e\left(C^{0}\right)+\chi_{1} e(Z)=\chi_{1} .
$$

By (3.9),

$$
n_{0, C}=P_{1, C}-0 . \chi_{0}=\chi_{1} .
$$

By Theorem [5] these are the only two BPS numbers: $n_{1, C}$ coming from $\mathcal{O}_{C}$ and $n_{0, C}$ coming from $p_{*} \mathcal{O}_{\bar{C}}$.

To see the geometry involved in the formulae for the local invariants, consider $P_{2}(C)$. The moduli space $P_{2}(C)$ is a $\mathbb{P}^{1}$-bundle over $\mathcal{M}_{2}(C) \cong C$. Hence,

$$
P_{2, C}=-2 P_{1, C} \text {. }
$$

Since $C$ is Gorenstein, we have the identification

$$
P_{n}(C) \cong \operatorname{Hilb}^{n}(C)
$$

by Proposition B.8 of Appendix B. Stratifying by both the number of points supported at the node $Z$ and by the type of the underlying sheaf (the subscript 0 denoting a line bundle, 1 the push-down of a line bundle from the normalisation), we expand the equality 3.19 as

$$
\begin{aligned}
-\chi_{0} e\left(S^{2} C^{0}\right)-\chi_{1} e\left(C^{0}\right) e(Z)-\chi_{0} e\left(\operatorname{Hilb}^{2}(C, Z)_{0}\right)- & \chi_{1} e\left(\operatorname{Hilb}^{2}(C, Z)_{1}\right) \\
& =-2\left(\chi_{0} e\left(C^{0}\right)+\chi_{1} e(Z)\right) .
\end{aligned}
$$

We use $\operatorname{Hilb}^{i}(C, Z)$ to denote the subset of $\operatorname{Hilb}^{i} C$ supported at $Z$. Since $C^{0} \cong \mathbb{C}^{*}$, $e\left(S^{i}\left(C^{0}\right)\right)=0$ for all $i$, and $Z$ is a single point. We obtain

$$
-\chi_{0} e\left(\operatorname{Hilb}^{2}(C, Z)_{0}\right)-\chi_{1} e\left(\operatorname{Hilb}^{2}(C, Z)_{1}\right)=-2 \chi_{1} .
$$

As in Section 3.5. the equation can be considered as two equations holding independently for $\chi_{0}$ and $\chi_{1}$ by applying Serre duality over the appropriate orbit $O_{i} \subset \mathcal{M}_{2}(C)$ instead of the whole moduli space. Then, we find

$$
e\left(\operatorname{Hilb}^{2}(C, Z)_{0}\right)=0 \text { and } e\left(\operatorname{Hilb}^{2}(C, Z)_{1}\right)=2 .
$$

Similarly, $P_{n}(C)$ is a $\mathbb{P}^{n-1}$-bundle over $C$, so $P_{n, C}=(-1)^{n-1} n P_{1, C}$. We obtain the following generalisation of $(3.20)$ :

$$
e\left(\operatorname{Hilb}^{n}(C, Z)_{0}\right)=0 \quad \text { and } \quad e\left(\operatorname{Hilb}^{n}(C, Z)_{1}\right)=n \quad \forall n \geq 1 .
$$

These consequences of Serre duality are what ensure that there are no further BPS numbers. In such a simple case, they can be verified directly. Since $C$ has 2-dimensional Zariski tangent space at $Z$,

$$
\operatorname{Hilb}^{2}(C, Z) \cong \mathbb{P}^{1},
$$

with two distinguished points $\{0, \infty\}$ corresponding to the directions of the two branches of $C$ at $Z$. Then,

$$
\operatorname{Hilb}^{2}(C, Z)_{0}=\mathbb{P}^{1} \backslash\{0, \infty\} \cong \mathbb{C}^{*} \text { and } \operatorname{Hilb}^{2}(C, Z)_{1}=\{0, \infty\},
$$

implying (3.20). Similarly, $\operatorname{Hilb}^{n}(C, Z)_{1}$ is $n$ points, corresponding to the $n$ different ways of distributing $(n-1)$ points between the two points in $\bar{C}=\mathbb{P}^{1}$ that lie over $Z$. Finally, since $\operatorname{Hilb}^{n}(C, Z)_{0}$ admits a $\mathbb{C}^{*}$-action without fixed points, we recover (3.21). 
In general, it is much harder to obtain the required results without Serre duality and the BPS generating function formalism. Even when the singularity has a $\mathbb{C}^{*}$ action, localisation is of limited use as it is hard to know on which orbit $O_{i}$ a fixed point lies.

Next, we consider curves with more nodes. Fix $C$ with arithmetic genus $g$, geometric genus $g-r$, and $r$ nodes. Let $i=0, \ldots, 2^{r-1}$ enumerate all of the partial normalisations

$$
p_{i}: \bar{C}_{i} \rightarrow C
$$

of $C$ including the identity $p_{0}$. These also enumerate the orbits of $\operatorname{Pic}(C)$ on the moduli spaces of sheaves $\mathcal{M}_{n}(C)$, where $O_{i}$ corresponds to push-downs $p_{i *}$ of line bundles from $C_{i}$.

Stable pairs can also be pushed down from a partial normalisation $\bar{C}_{i}$. Given a stable pair on $\bar{C}_{i}$,

$$
\mathcal{O}_{\bar{C}_{i}} \stackrel{s}{\rightarrow} L,
$$

we can push down $L$ and $s$ and compose with the canonical section of $p_{i *} \mathcal{O}_{\bar{C}_{i}}$,

$$
\mathcal{O}_{C} \rightarrow p_{i *} \mathcal{O}_{\bar{C}_{i}} \stackrel{p_{i *} s}{\longrightarrow} p_{i *} L,
$$

to give a stable pair on $C$. Let $g_{i}$ denote the arithmetic genus of $\bar{C}_{i}$, and let $\bar{C}_{i}^{0} \subset \bar{C}_{i}$ be the nonsingular locus. We see that

$$
S^{n-1+g_{i}}\left(\bar{C}_{i}^{0}\right) \subset P_{n}(C)
$$

by the push-down construction.

In fact, by (3.21) applied to both $C$ and the $\bar{C}_{i}$, the only parts of the stratification (by $O_{i}$ orbit type) of the moduli space $P_{n}(C)$ of stable pairs that contribute to $P_{n, C}$ are

$$
S^{n-1+g}\left(C^{0}\right) \sqcup \coprod_{i} S^{n-1+g_{i}}\left(\bar{C}_{i}^{0}\right) .
$$

The $i$ th stratum contributes with constant $\chi^{B}$-function $(-1)^{n-1} \chi_{i}$. The Euler characteristic of the nonsingular locus $C^{0}$ equals $e\left(\Sigma_{g}\right)$ and

$$
e\left(S^{j} C^{0}\right)=e\left(S^{j} \Sigma_{g}\right) \text {. }
$$

Similarly

Therefore, (3.22) yields

$$
e\left(S^{j} \bar{C}_{i}^{0}\right)=e\left(S^{j} \Sigma_{g_{i}}\right)
$$

$$
P_{n, C}=(-1)^{n-1}\left(\chi_{0} e\left(S^{n-1+g}\left(\Sigma_{g}\right)\right)+\sum_{i} \chi_{i} e\left(S^{n-1+g_{i}}\left(\Sigma_{g_{i}}\right)\right)\right) .
$$

Since the $i$ th term is the same as the contribution of $\pm \chi_{i}$ genus $g_{i}$ nonsingular curves in $X$, we see that

$$
n_{h, C}=\sum_{i: g_{i}=h}(-1)^{g_{i}} \chi_{i} .
$$

Proposition 3.23. $n_{h, C}=(-1)^{r} \sum_{i: g_{i}=h} \chi_{i}$.

Hence, the contributions of $C$ to $n_{h}$ come from all of the partial normalisations of $C$. Proposition 3.23 is closely related to an early interpretation of the GopakumarVafa invariants $n_{h}$ as the number of $h$-dimensional tori in the moduli spaces $\mathcal{M}_{n}(C)$ of sheaves. The tori may be viewed to be the orbits $O_{i}$, i.e., the (push-downs of) Jacobians of the curves $\bar{C}_{i}$. 


\section{Appendix A. Reduced CuRve Classes}

The methods of Section 1 do not extend to reduced classes $\beta$ since the sheaf $F$ underlying a stable pair $(F, s)$ need not be stable. For instance, $F$ could be reducible with nonscalar automorphisms. Hence, viewing stable pairs via the underlying sheaves decorated with a section is no longer profitable.

However, the local approach of Section 3 viewing pairs via their cokernels, works much the same as in the irreducible case. In fact, since the singularities $Z \subset C$ of any reduced curve also appear, locally analytically, in an irreducible curve, the proof of Theorem 5 goes through almost unchanged. We require the analogue of Theorem 4 for reduced reducible curves and check the invariance of $\chi^{B}$ under dualisation (2.6).

Theorem 6. Let $(F, s)$ be a stable pair on $X$, and let $L$ be a degree $\ell$ line bundle on the support of $F$. Then,

$$
\chi_{P}(F, s)=(-1)^{\ell} \chi_{P}(F \otimes L, t), \quad \chi_{P}(F, s)=\chi_{P}\left(\mathscr{E} x t^{2}\left(F, K_{X}\right), t\right),
$$

whenever the sections $t$ on the right define stable pairs.

Proof. The result can be proved for arbitrary stable pairs; the details will appear in a future paper [28. For the application to reduced $\beta$, we only need the result for stable pairs with reduced support. So, we work locally about a pair $(F, s)$ with support given by a reduced curve $C$ with $m$ irreducible components.

Just as in the proof of Theorem 4 there exists a smooth local divisor $D_{C}$ which intersects each irreducible component of $C$ transversally in a single smooth point of $C$, disjoint from the zeros of $s$. Hence, $D_{C}$ also intersects all nearby curves $C^{\prime}$ in $m$ smooth points.

Let $k \gg 0$. Tensoring sheaves $F$ with $\mathcal{O}\left(k D_{C}\right)$ and multiplying their sections $s$ by the canonical section $s_{D_{C}}^{k}$ of $\mathcal{O}\left(k D_{C}\right)$ yields a local embedding,

$$
P_{n}(X, \beta) \supset V_{n} \stackrel{\phi}{\longleftrightarrow} V_{n+m k} \subset P_{n+m k}(X, \beta) .
$$

Here, $V_{r}$ is a sufficiently small analytic neighbourhood of the locus $P_{r}(C) \subset P_{r}(X, \beta)$ of stable pairs supported on $C$.

By making $V_{n+m k}$ smaller if necessary, $\phi$ admits a left inverse

$$
V_{n} \stackrel{\psi}{\longleftarrow} V_{n+m k}
$$

given by forgetting the $m k$ points close to $D_{C}$. More precisely, in a neighbourhood of $D_{C}$ we replace any pair with support $C^{\prime}$ by the trivial pair $\left(\mathcal{O}_{C^{\prime}}, 1\right)$. Since these are smooth points of $C^{\prime}$, the map $\psi$ is locally a smooth fibre bundle with fibre the $(m k)$-th symmetric product of an open set of a smooth curve.

By relation (1.5), $\phi$ and $\psi$ simply multiply $\chi^{B}$-functions by $(-1)^{m k}$. Therefore, replacing $(F, s)$ by the image $\left(F\left(k D_{C}\right), s_{i} \cdot s^{k} D_{C}\right)$ under $\phi$, we may assume that $F$ has no higher cohomology.

We first explain how to prove Theorem [ 6 in case $L$ is trivial. Let $Q_{n}(X, \beta)$ denote the moduli space of triples $\left(F, s_{1}, s_{2}\right)$, where

- $F$ is a pure sheaf with 1-dimensional support and Hilbert polynomial $\chi(F(k))=\int_{\beta} c_{1}(\mathcal{O}(k))+n$,

- $s_{1}, s_{2} \in H^{0}(F)$ such that $\mathcal{O}_{X} \oplus \mathcal{O}_{X} \stackrel{\left(s_{1}, s_{2}\right)}{\longrightarrow} F$ has finite cokernel, 
modulo the obvious equivalence induced by automorphisms of $F$. While the corresponding moduli functor is easily seen to be separated and proper (and these triples have no automorphisms), the moduli space does not seem to have been constructed by GIT in the literature, though some very similar moduli problems have been treated in [11, 21]. Recently, Schmitt 29] has constructed just such a moduli space of torsion free sheaves, and his methods certainly extend to the pure sheaves above. Further details will appear in 28.

Let $U \subset Q_{n}(X, \beta)$ denote the open set on which both sections $s_{i}$ have finite cokernel. There are two projections from $U$ to $P_{n}(X, \beta)$, mapping $\left(F, s_{1}, s_{2}\right)$ to $\left(F, s_{1}\right)$ and $\left(F, s_{2}\right)$. Both maps are smooth with fibre an open set in $H^{0}(F)$ of dimension $n$ since $H^{1}(F)=0$. By Proposition 1.5(i) of [3],

$$
\chi_{P}\left(F, s_{1}\right)=(-1)^{n} \chi_{Q}\left(F, s_{1}, s_{2}\right)=\chi_{P}\left(F, s_{2}\right),
$$

as required.

Consider now $F \otimes L$ for some degree 0 line bundle $L$ defined in a neighbourhood of the support $C$ of $F$. Since $L$ can be trivialised over any finite number of points of $C$, we can write

$$
L \cong \mathcal{O}\left(D_{1}-D_{2}\right)
$$

for effective locally defined Cartier divisors $D_{i}$ intersecting $C$ transversally in points disjoint from the singularities of $C$ and the cokernel of the sections $s_{i}$. Let $d$ denote the degree of $D_{1}$ (and $\left.D_{2}\right)$ on $C$.

By the same working as in (A.1), the $\chi^{B}$-functions of

$$
(F, s) \text { and }\left(F\left(D_{1}+k D_{C}\right), s \cdot s_{D_{1}} \cdot s_{D_{C}}^{k}\right)
$$

differ only by $(-1)^{d+m k}$. A similar result holds for

$$
(F \otimes L, t) \quad \text { and } \quad\left(F \otimes L\left(D_{2}+k D_{C}\right), t \cdot s_{D_{2}} \cdot s_{D_{C}}^{k}\right) .
$$

By construction,

$$
F\left(D_{1}+k D_{C}\right)=F \otimes L\left(D_{2}+k D_{C}\right) .
$$

Then, the $\chi^{B}$-functions of (A.2) and A.3 agree by the previous proven case.

For $F \otimes L$, where the degree $L$ is nonzero, we apply A.1 separately to both $F$ and $F \otimes L$ and reduce to the degree 0 case.

Finally, consider $\mathscr{E} x t^{2}\left(F, K_{X}\right)$. Here, we form a moduli space of triples $\left(F, s_{1}, s_{2}\right)$, where

- $F$ is a pure sheaf with 1-dimensional support and Hilbert polynomial $\chi(F(k))=\int_{\beta} c_{1}(\mathcal{O}(k))+n$,

- $s_{1} \in H^{0}(F(r))$ has finite cokernel,

- $s_{2} \in H^{0}\left(\mathscr{E} x t^{2}\left(F, K_{X}\right)(r)\right)$ has finite cokernel,

and $r$ is a fixed integer.

The corresponding problem is separated (but not proper) and also has a quasiprojective moduli space which has never been explicitly written down in the literature. The moduli space maps to $P_{r+n}(X, \beta)$ by forgetting $s_{2}$ and to $P_{r-n}(X, \beta)$ by forgetting $s_{1}$. These maps are smooth fibrations of relative dimensions $r-n$ and $r+n$ respectively for $r \gg 0$. Hence, the $\chi^{B}$-function of the triple $\left(F, s_{1}, s_{2}\right)$ equals both

$$
(-1)^{r-n} \chi_{P}\left(F(r), s_{1}\right) \text { and }(-1)^{r+n} \chi_{P}\left(\mathscr{E} x t^{2}\left(F, K_{X}\right)(r), s_{2}\right) .
$$

The required result is then obtained from previous cases. 
The proof of Theorem 5 now applies verbatim to give an expression

$$
Z_{C}(q)=\sum_{s=g(\bar{C})}^{g} m_{s, C} q^{1-s}(1+q)^{2 s-2},
$$

as before, proving Theorems 1 and 2 for reduced curve classes.

However, there is a difference now. For a reducible curve,

$$
g(\bar{C})=1-e(\bar{C}) / 2
$$

might be negative. Here, the Gopakumar-Vafa conjecture takes the form of a sum over decompositions of $C$ into reducible components 9

$$
Z_{C}(q)=\sum_{\sum_{i} C_{i}=C} \sum_{r_{i}=0}^{g_{i}} \prod_{i}\left(n_{r_{i}, C_{i}} q^{1-r_{i}}(1+q)^{2 r_{i}-2}\right) .
$$

To properly define the BPS counts of $C$, we would need to show that all of the negative genus contributions of A.4 can be written inductively as products of BPS counts of lower degree curves. We currently have only partial results in this direction.

\section{Appendix B. Stable pairs on Gorenstein curves}

B.1. Gorenstein curves. The scheme-theoretic support $C$ of a stable pair $(F, s)$ is always Cohen-Macaulay. Equivalently, the dualising complex of $C$ is a sheaf $\omega_{C}$. If $\omega_{C}$ is a line bundle, then the curve is Gorenstein. Plane curves are basic examples. If $C$ can be embedded in a nonsingular surface $S$, then

$$
\left.\omega_{C} \cong \omega_{S}(C)\right|_{C},
$$

where $\omega_{S}$ is the canonical line bundle of $S$. Hence, $C$ is Gorenstein.

Let $(F, s) \in P_{1-g+n}(C)$ be a stable pair supported on a Gorenstein curve $C$. Dualising

$$
\mathcal{O}_{C} \stackrel{s}{\longrightarrow} F
$$

on $C$ yields the map

$$
F^{*} \longrightarrow \mathcal{O}_{C} .
$$

We will show that (B.1) is the ideal sheaf of a length $n$ subscheme $Z \subset C$. Conversely, we associate a stable pair to each subscheme $[Z] \in \operatorname{Hilb}^{n}(C)$ and establish a bijection

$$
P_{1-g+n}(C) \leftrightarrow \operatorname{Hilb}^{n}(C) .
$$

In Appendix B.2, the corresponding isomorphism of schemes is established for curves $C$ in surfaces.

Lemma B.2. Let $C$ be a Gorenstein curve, and let $F$ be a sheaf on $C$ which is generically locally free. Then, $F$ is pure if and only if

$$
\mathscr{E} x t_{C}^{i}\left(F, \mathcal{O}_{C}\right)=0 \quad \forall i>0 .
$$

\footnotetext{
${ }^{9}$ The $C_{i}$ have maximal arithmetic genus $g_{i}$, but need not be irreducible. We do not sum over different orderings of the $C_{i}$.
} 
Proof. Suppose $F$ is pure. Since $F$ is generically locally free, the sheaf $\mathscr{E} x t_{C}^{i}\left(F, \mathcal{O}_{C}\right)$ is supported at a finite number of points. Hence, the vanishing

$$
H^{0}\left(\mathscr{E} x t_{C}^{i}\left(F, \mathcal{O}_{C}\right) \otimes \mathcal{L}\right)=0 \quad \forall i>0
$$

for any line bundle $\mathcal{L}$ on $C$ implies the lemma.

Using the Gorenstein condition on $C$, let $\mathcal{L}=\omega_{C} \otimes L$. For $L \gg 0$, by the local-to-global spectral sequence and vanishing, (B.3) is isomorphic to

$$
\operatorname{Ext}_{C}^{i}\left(F \otimes L^{*}, \omega_{C}\right) \cong H^{1-i}\left(F \otimes L^{*}\right)^{*},
$$

using Serre duality. But $L^{*} \ll 0$ and $F$ is pure, so $H^{0}\left(F \otimes L^{-1}\right)=0$. Thus $H^{1-i}\left(F \otimes L^{-1}\right)=0$ for all $i>0$.

Conversely, suppose $F$ is generically locally free on $C$ with vanishing $\mathscr{E} x t^{1}\left(F, \mathcal{O}_{C}\right)$. Consider the sequence

$$
0 \rightarrow K \rightarrow F \rightarrow E \rightarrow 0,
$$

where $K$ is the largest subsheaf of $F$ with 0 -dimensional support. The quotient $E$ is pure and generically locally free. We have proven above the vanishing

$$
\mathscr{E} x t^{i}\left(E, \mathcal{O}_{C}\right)=0 \quad \forall i>0 .
$$

By (B.4), we conclude that

$$
\mathscr{E} x t^{1}\left(K, \mathcal{O}_{C}\right)=\mathscr{E} x t^{1}\left(F, \mathcal{O}_{C}\right),
$$

which vanishes by assumption. Hence,

$$
0=H^{0}\left(\mathscr{E} x t^{1}\left(K, \mathcal{O}_{C}\right)\right)=\operatorname{Ext}^{1}\left(K \otimes \omega_{C}, \omega_{C}\right)=H^{0}\left(K \otimes \omega_{C}\right)^{*}
$$

by Serre duality. Since $K$ has 0 -dimensional support, $K$ must vanish. Therefore, $F$ is pure.

Proposition B.5. A stable pair supported on a Gorenstein curve $C$ is equivalent to a 0-dimensional subscheme of $C$. Under the equivalence, the pair

$$
0 \rightarrow \mathcal{O}_{C} \stackrel{s}{\rightarrow} F \rightarrow Q \rightarrow 0
$$

is associated to the subscheme

$$
\mathcal{O}_{C} \cong \mathscr{E} x t^{0}\left(\mathcal{O}_{C}, \mathcal{O}_{C}\right) \rightarrow \mathscr{E} x t^{1}\left(Q, \mathcal{O}_{C}\right) \rightarrow 0 .
$$

Proof. Given a stable pair $(F, s) \in P_{1-g+n}(C)$, we apply $\mathscr{H}_{0} m_{C}\left(\cdot, \mathcal{O}_{C}\right)$ to the sequence (B.6). By the purity of $\mathcal{O}_{C}$ and LemmaB.2, we obtain the exact sequence

$$
0 \rightarrow F^{*} \rightarrow \mathcal{O}_{C} \rightarrow \mathscr{E} x t^{1}\left(Q, \mathcal{O}_{C}\right) \rightarrow 0 .
$$

Hence, $F^{*}$ is an ideal sheaf and $\mathscr{E} x t^{1}\left(Q, \mathcal{O}_{C}\right)$ is isomorphic to the structure sheaf of a subscheme of $C$. The higher terms in the sequence yield the vanishing of $\mathscr{E} x t^{\geq 2}\left(Q, \mathcal{O}_{C}\right)$, so $\mathscr{E} x t^{1}\left(Q, \mathcal{O}_{C}\right)$ has length $n$. We have defined a map of sets from $P_{1-g+n}(C)$ to $\operatorname{Hilb}^{n}(C)$.

Given a subscheme $[Z] \in \operatorname{Hilb}^{n}(C)$, the dual of the exact sequence

$$
0 \rightarrow \mathscr{I}_{Z} \rightarrow \mathcal{O}_{C} \rightarrow \mathcal{O}_{Z} \rightarrow 0
$$

starts as

$$
0 \rightarrow \mathcal{O}_{C} \rightarrow \mathscr{I}_{Z}^{*} \rightarrow \mathscr{E} x t^{1}\left(\mathcal{O}_{Z}, \mathcal{O}_{C}\right) \rightarrow 0 .
$$

By Lemma B.2, the higher terms in the sequence yield the vanishing of $\mathscr{E} x t^{\geq 2}\left(O_{Z}, \mathcal{O}_{C}\right)$, so $\mathscr{E} x t^{1}\left(\mathcal{O}_{Z}, \mathcal{O}_{C}\right)$ has length $n$ and $\mathscr{I}_{Z}^{*}$ has holomorphic Euler characteristic $1-g+n$. 
Since $\mathscr{E} x t^{\geq 1}\left(\mathscr{I}_{Z}, \mathcal{O}_{C}\right)$ vanishes by Lemma B.2 $\mathscr{I}_{Z}^{*}=R \mathscr{H} o m\left(\mathscr{I}_{Z}, \mathcal{O}_{C}\right)$. By applying $R \mathscr{H} o m\left(\cdot, \mathcal{O}_{C}\right)$ again, we obtain

$$
\operatorname{R} \mathscr{H} o m\left(\mathscr{I}_{Z}^{*}, \mathcal{O}_{C}\right)=\mathscr{I}_{Z} \text {. }
$$

Therefore, $\mathscr{E} x t^{\geq 1}\left(\mathscr{I}_{Z}^{*}, \mathcal{O}_{C}\right)$ vanishes and $\mathscr{I}_{Z}^{*}$ is pure by Lemma B.2. We conclude that

$$
\mathcal{O}_{C} \rightarrow \mathscr{I}_{Z}^{*}
$$

determines a stable pair in $P_{1-g+n}(C)$. We have defined a map of sets from $\operatorname{Hilb}^{n}(C)$ to $P_{1-g+n}(C)$.

As the two constructions are easily seen to be inverse to each other, a bijection is established.

Simple examples where the quotient $Q$ of $($ B.6. $)$ fails to be a structure sheaf can be found on nodal curves. However, $\mathscr{E} x t^{1}\left(Q, \mathcal{O}_{C}\right)$ is always a structure sheaf. For the equivalence of Proposition B.5, the duals are necessary.

The equivalence of Proposition B.5 is continuous. For Gorenstein curves, the moduli of pairs $P_{1-g+n}(C)$ is homeomorphic as a topological space to the Hilbert scheme Hilb $^{n}(C)$. If $C$ is also reduced, we can apply the result of Proposition 3.13. and the method of Theorem 5 replacing the $\chi^{B}$-function by $(-1)^{\mathrm{dim}}$. The result is that the generating function of Euler characteristics

$$
\sum_{i=0}^{\infty} e\left(\operatorname{Hilb}^{i}(C)\right) q^{i}
$$

can be written in the form

$$
\sum_{r=g(\bar{C})}^{g} n_{r, C} q^{g-r}(1-q)^{2 r-2}
$$

for integers $n_{r, C}$, where $r$ runs from the geometric genus $g(\bar{C})$ to the arithmetic genus $g=g(C)$ of $C$.

B.2. Surfaces. Let $S$ be a nonsingular projective surface, and let $\beta \in H_{2}(S, \mathbb{Z})$. Let $\mathcal{M}$ denote the moduli space of pure dimension 1 subschemes of $S$ in class $\beta$. Since the holomorphic Euler characteristic of such subschemes is determined by adjunction, $\mathcal{M}$ is a Hilbert scheme of curves. Let

$$
\mathcal{C} \subset S \times \mathcal{M}
$$

denote the universal curve.

By Proposition B.5. $P_{1-g+n}(S, \beta)$ is in bijective correspondence with the relative Hilbert scheme $\operatorname{Hilb}^{n}(\mathcal{C} / \mathcal{M})$, where

$$
2 g-2=\int_{S} K_{S} \cdot \beta+\beta^{2} .
$$

We now show that the correspondence is an isomorphism of schemes.

Proposition B.8. The moduli space of pairs $P_{1-g+n}(S, \beta)$ is isomorphic to the relative Hilbert scheme $\operatorname{Hilb}^{n}(\mathcal{C} / \mathcal{M})$.

Proof. Let $P$ denote the space of pairs $P_{1-g+n}(S, \beta)$ and let $(\mathbb{F}, s)$ denote the universal stable pair on $S \times P$. The kernel of the canonical map

$$
\mathcal{O}_{S \times P} \stackrel{s}{\longrightarrow} \mathbb{F}
$$


is the ideal sheaf of a relative curve $\mathcal{D}$ flat over $P$. By the universal property of $\mathcal{M}$, we obtain a map

$$
f: P \rightarrow \mathcal{M}
$$

such that $\mathcal{D}=\left(1_{S} \times f\right)^{*} \mathcal{C}$ is the pull-back of the universal curve.

We have the canonical exact sequence

$$
0 \rightarrow \mathcal{O}_{\mathcal{D}} \stackrel{s}{\longrightarrow} \mathbb{F} \rightarrow \mathbb{Q} \rightarrow 0,
$$

with the universal quotient $\mathbb{Q}$ also flat over $P$. Applying $\mathscr{H}_{o} m_{\mathcal{D}}\left(\cdot, \mathcal{O}_{\mathcal{D}}\right)$ yields

$$
0 \rightarrow \mathbb{F}^{*} \rightarrow \mathcal{O}_{\mathcal{D}} \rightarrow \mathscr{E} x t^{1}\left(\mathbb{Q}, \mathcal{O}_{\mathcal{D}}\right) \rightarrow \mathscr{E} x t^{1}\left(\mathbb{F}, \mathcal{O}_{\mathcal{D}}\right)
$$

The last term vanishes by flatness, base change, and Lemma B.2. Similarly $\mathscr{E} x t^{\geq 2}\left(\mathbb{F}, \mathcal{O}_{\mathcal{D}}\right)=0$, which implies $\mathscr{E} x t^{\geq 2}\left(\mathbb{Q}, \mathcal{O}_{\mathcal{D}}\right)=0$. Therefore $\mathscr{E} x t^{1}\left(\mathbb{Q}, \mathcal{O}_{\mathcal{D}}\right)$ is flat over $P$ with relative length $n$.

By the universal property of $\operatorname{Hilb}^{n}(\mathcal{C} / \mathcal{M})$, the quotient sequence $\mathrm{B} .9$ defines a map from $P$ to $\operatorname{Hilb}^{n}(\mathcal{C} / \mathcal{M})$ through which $f$ factors. The inverse map is similarly a relative version of the map in the proof of Proposition B.5.

\section{Appendix C. $K 3$ surfaces}

C.1. Nonsingularity. Interesting irreducible classes on Calabi-Yau 3-folds can be found on $K 3$ fibrations. Let

$$
\pi: X \rightarrow \triangle
$$

be a fibration of a Calabi-Yau 3-fold over a nonsingular curve satisfying the following properties:

(i) the fibres of $\pi$ are $K 3$ surfaces,

(ii) $S=\pi^{-1}(0)$ carries an irreducible $(1,1)$-class $\beta \in H_{2}(S, \mathbb{Z})$,

(iii) $\pi$ is transverse to the Noether-Lefschetz locus associated to $\beta$.

For the above geometry, we do not require $X$ to be compact.

Condition (iii) is equivalent to requiring the Kodaira-Spencer class

$$
\kappa \in H^{1}(S, T S) \cong H^{1,1}(S)
$$

to evaluate to something nonzero on $\beta$. By (iii), a curve on $S$ in class $\beta$ does not deform, even to first order, away from $S$. Therefore,

$$
P_{n}(S, \beta) \rightarrow P_{n}\left(X, \iota_{*} \beta\right)
$$

is a bijective correspondence with a component of the moduli space of pairs, where

$$
\iota: S \rightarrow X
$$

We will show that C.1 is a local isomorphism of schemes in Lemma C.7 below.

The moduli space $P_{n}(S, \beta)$ is empty if $n<1-g$, where

$$
2 g-2=\int_{S} \beta^{2}
$$

as before.

Proposition C.2. For $\beta$ irreducible, the moduli space $P_{n}(S, \beta)$ is nonsingular of dimension $n+2 g-1$. 
Proof. We briefly recall the deformation theory of pairs on surfaces $9,20,21$. The deformations of the pair $\mathcal{O}_{S} \rightarrow F$ are governed by $\operatorname{Hom}\left(I^{\bullet}, F\right)$ and the obstruction theory by $\operatorname{Ext}^{1}\left(I^{\bullet}, F\right)$. The maps

$$
\operatorname{Ext}^{1}\left(I^{\bullet}, F\right) \rightarrow \operatorname{Ext}^{2}(F, F) \stackrel{\operatorname{tr}}{\longrightarrow} H^{2}\left(\mathcal{O}_{S}\right)
$$

take the obstructions to deforming $\mathcal{O}_{S} \rightarrow F$ first to the obstructions to deforming the sheaf $F$ and then 25 to the obstruction to deforming the determinant $\mathcal{O}_{S}(C)$ of the sheaf $F$, where $C$ is the support of $F$. Since the Picard scheme is smooth, the latter obstruction vanishes. Hence, the obstructions to deforming $F$ can be taken to lie in the trace-free group $\operatorname{Ext}^{2}(F, F)_{0}$, and the obstructions to deforming the pair $\mathcal{O}_{S} \rightarrow F$ lie in the kernel of the map

$$
\operatorname{Ext}^{1}\left(I^{\bullet}, F\right) \rightarrow H^{2}\left(\mathcal{O}_{S}\right)
$$

obtained from (C.3). If the kernel is 0 , then by the analysis in [9, 20, 21, the moduli space is nonsingular.

The deformation and obstruction spaces sit inside the exact sequence

$$
\begin{aligned}
& 0 \rightarrow \operatorname{Hom}(F, F) \rightarrow H^{0}(F) \rightarrow \operatorname{Hom}\left(I^{\bullet}, F\right) \rightarrow \operatorname{Ext}^{1}(F, F) \rightarrow \\
& H^{1}(F) \rightarrow \operatorname{Ext}^{1}\left(I^{\bullet}, F\right) \rightarrow \operatorname{Ext}^{2}(F, F) \rightarrow 0,
\end{aligned}
$$

induced by the triangle $I \bullet \rightarrow \mathcal{O}_{S} \rightarrow F$. We claim that the first arrow on the second line is zero, or equivalently, that

$$
\operatorname{Ext}^{1}(F, F) \rightarrow H^{1}(F)
$$

is onto. It is enough to show that the composition

$$
H^{1}\left(\mathcal{O}_{C}\right) \stackrel{\text { id }}{\longrightarrow} H^{1}(\mathscr{H} o m(F, F)) \subseteq \operatorname{Ext}^{1}(F, F) \rightarrow H^{1}(F)
$$

is onto. But (C.5) is multiplication by the section $s$, sitting in the exact sequence

$$
H^{1}\left(\mathcal{O}_{C}\right) \stackrel{s}{\rightarrow} H^{1}(F) \rightarrow H^{1}(Q) .
$$

Since the support of $Q$ is 0 -dimensional, $H^{1}(Q)$ vanishes.

The support $C$ of $F$ is reduced and irreducible since $C$ is Cohen-Macaulay and $\beta$ is irreducible. Since the rank of $F$ on $C$ is $1, F$ is simple. Therefore the identity map

$$
H^{0}\left(\mathcal{O}_{S}\right) \stackrel{\text { id }}{\longrightarrow} \operatorname{Hom}(F, F)
$$

is an isomorphism. By Serre duality and $K_{S} \cong \mathcal{O}_{S}$, the trace map

$$
\operatorname{Ext}^{2}(F, F) \stackrel{\operatorname{tr}}{\longrightarrow} H^{2}\left(\mathcal{O}_{S}\right)
$$

is also an isomorphism.

So (C.4) has become the sequences

$$
0 \rightarrow H^{0}(F) /\langle s\rangle \rightarrow \operatorname{Hom}\left(I^{\bullet}, F\right) \rightarrow \operatorname{Ext}^{1}(F, F) \rightarrow H^{1}(F) \rightarrow 0
$$

and

$$
\operatorname{Ext}^{1}\left(I^{\bullet}, F\right) \stackrel{\sim}{\longrightarrow} \operatorname{Ext}^{2}(F, F) \cong H^{2}\left(\mathcal{O}_{S}\right)=\mathbb{C} .
$$

Therefore, the obstruction space

$$
\operatorname{ker}\left(\operatorname{Ext}^{1}\left(I^{\bullet}, F\right) \rightarrow H^{2}\left(\mathcal{O}_{S}\right)\right)
$$

vanishes, and the moduli space is nonsingular. 
The dimension can be easily computed. The space of curves of class $\beta$ has dimension $g$. For a nonsingular curve $C$, the dimension of the space of pairs $P_{n}(C)$ is $n+g-1$. Hence, the dimension of $P_{n}(S, \beta)$ is $n+2 g-1$.

Proposition C.2 was first proven by Kawai-Yoshioka in [17, where the space of stable pairs on a surface is interpreted as a moduli space of D2-D0 branes. We include the above argument for completeness here. With hindsight, one can see many of the techniques we use in three dimensions in the 2-dimensional analysis of [17.

Lemma C.7. For $\beta$ irreducible, $P_{n}(S, \beta) \subset P_{n}\left(X, \iota_{*} \beta\right)$ is isomorphic to a component of the moduli space of pairs.

Proof. Let $I_{X}^{\bullet}=\left\{\mathcal{O}_{X} \rightarrow \iota_{*} F\right\}$ and $I_{S}^{\bullet}=\left\{\mathcal{O}_{S} \rightarrow F\right\}$. There is a canonical exact triangle

$$
F(-S) \rightarrow L \iota^{*}\left(I_{X}^{\bullet}\right) \rightarrow I_{S}^{\bullet}
$$

on $S$. Applying $\operatorname{Hom}_{S}(\cdot, F)$ gives

$$
\begin{aligned}
0 \rightarrow \operatorname{Hom}_{S}\left(I_{S}^{\bullet}, F\right) \rightarrow \operatorname{Hom}_{X}\left(I_{X}^{\bullet}, \iota_{*} F\right) & \rightarrow \operatorname{Hom}_{S}(F, F(S)) \\
& \rightarrow \operatorname{Ext}_{S}^{1}\left(I_{S}^{\bullet}, F\right) \rightarrow \operatorname{Ext}_{X}^{1}\left(I_{X}^{\bullet}, \iota_{*} F\right) \rightarrow \ldots .
\end{aligned}
$$

From the simplicity of $F$, we see that

$$
\operatorname{Hom}_{S}(F, F(S)) \cong H^{0}\left(\mathcal{O}_{S}(S)\right) .
$$

Using the identification (C.6), we can rewrite the above long exact sequence as

$$
0 \rightarrow \operatorname{Hom}_{S}\left(I_{S}^{\bullet}, F\right) \rightarrow \operatorname{Hom}_{X}\left(I_{X}^{\bullet}, \iota_{*} F\right) \rightarrow H^{0}\left(\mathcal{O}_{S}(S)\right) \rightarrow H^{2}\left(\mathcal{O}_{S}\right) \rightarrow \cdots .
$$

The map from $H^{0}\left(\mathcal{O}_{S}(S)\right) \cong T_{0} \triangle$ to $H^{2}\left(\mathcal{O}_{S}\right) \cong \mathbb{C}$ takes a normal direction to the fibre $S \subset X$ to the obstruction to deforming the determinant of $I^{\bullet}$ sideways. The determinant is $\mathcal{O}_{S}(-C)$, where $C$ is the support of $F$, and the obstruction to deforming sideways is $\langle\kappa, \beta\rangle \neq 0$. Therefore

$$
\operatorname{Hom}_{S}\left(I_{S}^{\bullet}, F\right) \cong \operatorname{Hom}_{X}\left(I_{X}^{\bullet}, \iota_{*} F\right) .
$$

All the deformations of the pair $\left(\iota_{*} F, \iota_{*} s\right)$ are the push-forwards of deformations of the pair $(F, s)$. By Proposition C.2 the moduli space of the latter is nonsingular. Hence, the moduli space of the former is also nonsingular and the push-forward map is a local isomorphism.

C.2. BPS states. Let $P_{n}(S, h)$ denote the moduli space for an irreducible class $\beta$ satisfying

$$
2 h-2=\int_{S} \beta^{2}
$$

Let $\Omega_{P}$ be the cotangent bundle of the moduli space $P_{n}(S, h)$. The self-dual obstruction theory on $P_{n}(S, h)$ induced from the inclusion (C.1) has obstruction bundle $\Omega_{P}$. Hence, the contribution of $P_{n}(S, h)$ to the invariants of the stable pairs of $X$ is

$$
Z_{h}^{S}(y)=\sum_{n}(-1)^{n+2 h-1} e\left(P_{n}(S, h)\right) y^{n}
$$


Fortunately, the topological Euler characteristics of $P_{n}(S, h)$ have been calculated by Kawai-Yoshioka. By Theorem 5.80 of [17,

$$
\begin{aligned}
\sum_{h=0}^{\infty} \sum_{n=1-h}^{\infty} e\left(P_{n}(S, h)\right) & y^{n} q^{h} \\
& =\left(\sqrt{y}-\frac{1}{\sqrt{y}}\right)^{-2} \prod_{n=1}^{\infty} \frac{1}{\left(1-q^{n}\right)^{20}\left(1-y q^{n}\right)^{2}\left(1-y^{-1} q^{n}\right)^{2}} .
\end{aligned}
$$

For our pairs' invariants, we require the signed Euler characteristics,

$$
\sum_{h=0}^{\infty} Z_{h}^{S}(y) q^{h}=\sum_{h=0}^{\infty} \sum_{n=1-h}^{\infty}(-1)^{n+2 h-1} e\left(P_{n}(S, h)\right) y^{n} q^{h} .
$$

Therefore, $\sum_{h=0}^{\infty} Z_{h}^{S}(y) q^{h}$ is

$$
-\left(\sqrt{-y}-\frac{1}{\sqrt{-y}}\right)^{-2} \prod_{n=1}^{\infty} \frac{1}{\left(1-q^{n}\right)^{20}\left(1+y q^{n}\right)^{2}\left(1+y^{-1} q^{n}\right)^{2}} .
$$

Let $r_{g, h}$ be the BPS invariant in genus $g$ and class $\beta$ satisfying (C.8). By the definition of the BPS invariants for the theory of stable pairs (2.9),

$$
\sum_{h=0}^{\infty} Z_{h}^{S}(y) q^{h}=\sum_{g=0}^{\infty} \sum_{h=0}^{\infty}(-1)^{g-1} r_{g, h}\left(\sqrt{-y}-\frac{1}{\sqrt{-y}}\right)^{2 g-2} q^{h} .
$$

Putting all the formulae together yields the following result.

Proposition C.9. We have

$$
\sum_{g=0}^{\infty} \sum_{h=0}^{\infty}(-1)^{g} r_{g, h}\left(\sqrt{z}-\frac{1}{\sqrt{z}}\right)^{2 g} q^{h}=\prod_{n=1}^{\infty} \frac{1}{\left(1-q^{n}\right)^{20}\left(1-z q^{n}\right)^{2}\left(1-z^{-1} q^{n}\right)^{2}} .
$$

Proposition C.9 is exactly the Katz-Klemm-Vafa [16 prediction for BPS state counts for irreducible classes on a $K 3$ surface. Our proof is really just an interpretation of the calculation of Kawai-Yoshioka in the theory of stable pairs.

If $\beta \in H_{2}(S, \mathbb{Z})$ is primitive, then the geometry can be deformed to make $\beta$ irreducible, and PropositionC.9 still applies. If $\beta$ is not primitive, the Katz-KlemmVafa formula prediction is not yet proven. On the Gromov-Witten side, the KatzKlemm-Vafa formula is open even in the primitive case. See 24 for a discussion.

C.3. Yau-Zaslow. We can specialize the Katz-Klemm-Vafa formula of Proposition C.9 to genus 0 by letting $z \rightarrow 1$,

$$
\sum_{h=0}^{\infty} r_{0, h} q^{h}=\prod_{n=1}^{\infty}\left(1-q^{n}\right)^{-24}
$$

Consider the linear system of curves on $S$ of irreducible class $\beta$ satisfying

$$
2 h-2=\int_{S} \beta^{2} .
$$

By Theorem [5, only curves of geometric genus 0 contribute to the BPS count $r_{0, h}$. By the main result of [6], the only genus 0 curves on a generic $K 3$ (with algebraic 
class $\beta$ ) are nodal. By Proposition 3.23, a nodal rational curve $C$ contributes

$$
(-1)^{0} \sum_{i: g_{i}=0} \chi_{i}=(-1)^{0}(-1)^{n-1} \chi_{P_{n}(C)}^{B}=(-1)^{n-1}(-1)^{n+2 g-1}=1
$$

to $r_{0, h}$.

We conclude the strong enumerative form of the Yau-Zaslow formula: $r_{0, h}$ exactly counts rational curves on a generic $K 3$ surface with $\beta$ algebraic.

\section{ACKNOWLEDGEMENTS}

We thank J. Bryan and D. Maulik for many conversations related to stable pairs and A. Marian for pointing out the basic connection to the work of Kawai-Yoshioka. We are grateful to S. Kleiman for advice on Jacobians of singular curves, and HuaLiang Chang for a careful reading of the manuscript.

\section{REFERENCES}

[1] A. Bayer, Polynomial Bridgeland stability conditions and the large volume limit, arXiv:0712.1083.

[2] A. Beauville, Counting rational curves on K3 surfaces, arXiv:alg-geom/9701019.

[3] K. Behrend, Donaldson-Thomas invariants via microlocal geometry, arXiv:alg-geom/ 0507523.

[4] K. Behrend and B. Fantechi, The intrinsic normal cone, Invent. Math., 128, 45-88, 1997. MR1437495 (98e:14022)

[5] J. Bryan and C. Leung, The enumerative geometry of K3 surfaces and modular forms, J. AMS, 13, 371-410, 2000. MR1750955 (2001i:14071)

[6] X. Chen, Singularities of rational curves on K3 surfaces, arXiv:alg-geom/9812050.

[7] R. Gopakumar and C. Vafa, M-theory and topological strings-I, arXiv:hep-th/9809187.

[8] R. Gopakumar and C. Vafa, M-theory and topological strings-II, arXiv:hep-th/9812127.

[9] M. He, Espaces de modules de systèmes cohérents, Internat. J. of Math., 9, 545-598, 1998. MR.1644040 (99i:14016)

[10] S. Hosono, M. Saito, and A. Takahashi, Relative Lefschetz action and BPS state counting, Internat. Math. Res. Notices, 15, 783-816, 2001. MR1849482 (2002c:14024)

[11] D. Huybrechts and M. Lehn, Framed modules and their moduli. Internat. J. Math., 6, 297324, 1995. MR1316305(96a:14017)

[12] D. Huybrechts and M. Lehn, The geometry of moduli spaces of sheaves. Aspects of Mathematics, E31. Friedr. Vieweg \& Sohn, Braunschweig, 1997. MR1450870 (98g:14012)

[13] D. Huybrechts and R. P. Thomas, Deformation-obstruction theory for complexes via Atiyah and Kodaira-Spencer classes, arXiv:0805.3527.

[14] D. Joyce, Configurations in abelian categories. IV. Invariants and changing stability conditions, arXiv:alg-geom/0410268.

[15] S. Katz, Genus zero Gopakumar-Vafa invariants of contractible curves, arXiv:alg-geom/ 0601193.

[16] S. Katz, A. Klemm, and C. Vafa, M-theory, topological strings and spinning black holes, Adv. Theor. Math. Phys., 3, 1445-1537, 1999. MR.1796683(2002b:81124)

[17] T. Kawai and K. Yoshioka, String partition functions and infinite products, Adv. Theor. Math. Phys., 4, 397-485, 2000. MR1838446 (2002g:11054)

[18] A. Klemm, D. Maulik, R. Pandharipande, and E. Scheidegger, Noether-Lefschetz theory and the Yau-Zaslow conjecture, arXiv:0807.2477.

[19] M. Kontsevich and Y. Soibelman, Stability structures, motivic Donaldson-Thomas invariants and cluster transformations, in preparation.

[20] J. Le Potier, Systèmes cohérents et structures de niveau, Astérisque, 214, 143, 1993. MR1244404 (95e:14005)

[21] J. Le Potier, Faisceaux semi-stables et systèmes cohérents, In Vector bundles in algebraic geometry (Durham,1993), Vol. 208 of London Math. Soc. Lecture Note Ser., 179-239. Cambridge Univ. Press: Cambridge, 1995. MR1338417 (96h:14010) 
[22] J. Li and G. Tian, Virtual moduli cycles and Gromov-Witten invariants of algebraic varieties, J. Amer. Math. Soc., 11, 119-174, 1998. MR1467172 (99d:14011)

[23] D. Maulik, N. Nekrasov, A. Okounkov, and R. Pandharipande, Gromov-Witten theory and Donaldson-Thomas theory. I, Compos. Math., 142, 1263-1285, 2006. arXiv:alggeom/0312059. MR2264664(2007i:14061)

[24] D. Maulik and R. Pandharipande, Gromov-Witten theory and Noether-Lefschetz theory, arxiv:0705.1653.

[25] S. Mukai, Symplectic structure of the moduli space of sheaves on an Abelian or K3 surface, Invent. Math., 77, 101-116, 1984. MR751133 (85j:14016)

[26] R. Pandharipande and R. P. Thomas, Curve counting via stable pairs in the derived category, arXiv:0707.2348.

[27] R. Pandharipande and R. P. Thomas, The 3-fold vertex via stable pairs, Geom. Topol., 13, 1835-1876, 2009. MR2497313

[28] R. Pandharipande and R. P. Thomas, in preparation.

[29] A. Schmitt, Geometric Invariant Theory and Decorated Principal Bundles, Zurich Lectures in Advanced Mathematics, Zurich, 2008. MR2437660

[30] A. Schwarz and I. Shapiro, Some remarks on Gopakumar-Vafa invariants, Pure Appl. Math. Quart., 1, 817-826, 2005. MR2201001 (2006k:14015)

[31] R. P. Thomas, A holomorphic Casson invariant for Calabi-Yau 3-folds, and bundles on K3 fibrations, J. Differential Geom., 54, 367-438, 2000. MR1818182 (2002b:14049)

[32] Y. Toda, Birational Calabi-Yau 3-folds and BPS state counting, arXiv:0707.1643.

[33] Y. Toda, Limit stable objects on Calabi-Yau 3-folds, arXiv:0803.2356.

[34] S-T. Yau and E. Zaslow, BPS states, string duality, and nodal curves on K3, Nucl. Phys. B471, 503-512, 1996. MR1398633 (97e:14066)

Department of Mathematics, Princeton University, Princeton, New Jersey 08544

E-mail address: rahulp@math.princeton.edu

Department of Mathematics, Imperial College, London, England

E-mail address: rpwt@imperial.ac.uk 